\title{
A vector equilibrium problem for symmetrically located point charges on a sphere
}

\author{
Juan G. Criado del Rey * Arno B.J. Kuijlaars ${ }^{\dagger}$
}

\begin{abstract}
We study the equilibrium measure on the two dimensional sphere in the presence of an external field generated by $r+1$ equal point charges that are symmetrically located around the north pole. The support of the equilibrium measure is known as the droplet. The droplet has a motherbody which we characterize by means of a vector equilibrium problem (VEP) for $r$ measures in the complex plane.

The model undergoes two transitions which is reflected in the support of the first component of the minimizer of the VEP, namely the support can be a finite interval containing 0 , the union of two intervals, or the full half-line. The two interval case corresponds to a droplet with two disjoint components, and it is analyzed by means of a genus one Riemann surface.
\end{abstract}

\section{Introduction}

\subsection{Equilibrium on the sphere}

This paper deals with an electrostatic equilibrium problem for free charges on the unit sphere $\mathbb{S}^{2} \subset \mathbb{R}^{3}$ with logarithmic interaction under the influence of a finite number of fixed point charges [7, 11, 16, 39. Suppose there are $r+1$ fixed charges at points $p_{0}, \ldots, p_{r}$ on $\mathbb{S}^{2}$, and each $p_{j}$ carries a charge $a_{j}>0$, leading to a charge distribution

$$
\sigma=\sum_{j=0}^{r} a_{j} \delta_{p_{j}} .
$$

*Department of Mathematics, Katholieke Universiteit Leuven, Belgium, Email: juan.gcriadodelrey@kuleuven.be. Supported by FWO Flanders project EOS 30889451.

${ }^{\dagger}$ Department of Mathematics, Katholieke Universiteit Leuven, Belgium, Email: arno.kuijlaars@kuleuven.be. Supported by long term structural funding-Methusalem grant of the Flemish Government, and by FWO Flanders projects EOS 30889451, G.0864.16 and G.0910.20. 
Then there exists an equilibrium measure $\mu_{\sigma}$ in the presence of the fixed charges that is the unique probability measure on $\mathbb{S}^{2}$ that satisfies for some constant $\ell$,

$$
\begin{array}{ll}
U^{\mu_{\sigma}}+U^{\sigma}=\ell, & \text { on } D_{\sigma}=\operatorname{supp}\left(\mu_{\sigma}\right), \\
U^{\mu_{\sigma}}+U^{\sigma} \geq \ell, & \text { on } \mathbb{S}^{2},
\end{array}
$$

where we use

$$
U^{\mu}(x)=\int \log \frac{1}{\|x-y\|} d \mu(y)
$$

to denote the logarithmic potential of a measure $\mu$. The domain $D_{\sigma}$ is known as the droplet, and it determines the measure $\mu_{\sigma}$ since

$$
\mu_{\sigma}=\left(\lambda\left(D_{\sigma}\right)\right)^{-1} \lambda_{D_{\sigma}}
$$

where $\lambda_{D}$ denotes the restriction to $D$ of the normalized Lebesgue measure $\lambda$ on the sphere. It is known that

$$
\lambda\left(D_{\sigma}\right)=\frac{1}{1+\sigma\left(\mathbb{S}^{2}\right)}=\frac{1}{1+\sum_{j=0}^{r} a_{j}} .
$$

see e.g. [11, Appendix A].

A motherbody (or a potential theoretic skeleton [25]) for $D_{\sigma}$ is a probability measure $\sigma^{*}$ supported on a one-dimensional subset of $\mathbb{S}^{2}$ (i.e., a curve, or a system of curves) such that for some constant $\ell^{*}$,

$$
\begin{array}{ll}
U^{\sigma^{*}}=U^{\mu_{\sigma}}+\ell^{*}, & \text { on } \mathbb{S}^{2} \backslash D_{\sigma}, \\
U^{\sigma^{*}} \geq U^{\mu_{\sigma}}+\ell^{*}, & \text { on } \mathbb{S}^{2} .
\end{array}
$$

Motherbodies are connected to a variety of topics in applied complex analysis, such as quadrature domains and Schwarz functions [1, 12, 24, 34], partial balayage and Hele-Shaw flows [23], orthogonal polynomials in the complex plane [3, 4, 38] and normal matrix models [5, 48].

The aim of this paper is to construct such a motherbody by means of a vector equilibrium problem in the special situation where the points are in a symmetric position around a distinguished point on the unit sphere, that without loss of generality we can take as the north pole. More precisely, we assume that the distance to the north pole is the same for each point $p_{j}$, which means that the points are on a circle of constant latitude. On this circle the points are evenly distributed, like vertices of a regular $r+1$-gon. We also assume

$$
a_{j}=a, \quad \text { for } j=0, \ldots, r
$$


In this situation we are able to compute the motherbody, which, because of rotational symmetry, is supported on $r+1$ meridians (lines of constant longitude) that connect the north and south poles. From the motherbody we go on to construct the droplet $D_{\sigma}$.

With fixed points $p_{0}, \ldots, p_{r}$, the droplet and the support of the motherbody decrease as we increase $a$. We find three possible situations and the transitions between them.

- For small $a>0$, the droplet is big and the complement $\mathbb{S}^{2} \backslash D_{\sigma}$ consists of $r+1$ disjoint spherical caps, one around each of the points $p_{j}$. The motherbody is supported on the full meridians with a positive density.

- For a first critical value $a_{1, c r}$, the spherical caps are tangent to each other. The density of the motherbody becomes zero at the points of tangency.

- For $a>a_{1, c r}$ the droplet is no longer the complement of disjoint spherical caps. For $a$ somewhat larger than $a_{1, c r}$ the droplet will have two connected components (provided $r \geq 2$ ), one containing the north pole and the other one the south pole. The motherbody is not fully supported anymore. On each meridian the support has two parts, one with the north pole and one with the south pole.

- For a second critical value $a_{2, c r}$ one of the components disappers. If the points $p_{j}$ are in the northern hemisphere, then the component containing the north pole disappears. Also the parts of the motherbody containing the north pole have disappeared at the second critical value.

- For larger $a>a_{2, c r}$ the droplet $D_{\sigma}$ is simply connected containing the south pole (assuming again that the points $p_{j}$ are in the northern hemisphere). The support of the motherbody consists of $r+1$ segments containing the south pole, one segment along each meridian.

- As $a \rightarrow \infty$, the droplet and the support of the motherbody further shrink to the south pole.

\subsection{The case $r=1$}

For $r=1$ the two spherical caps are tangent at the north pole at the critical value $a_{1, c r}$. Then there is no second critical $a$-value since for each $a>a_{1, c r}$ the droplet is simply connected. The support of the motherbody is an 

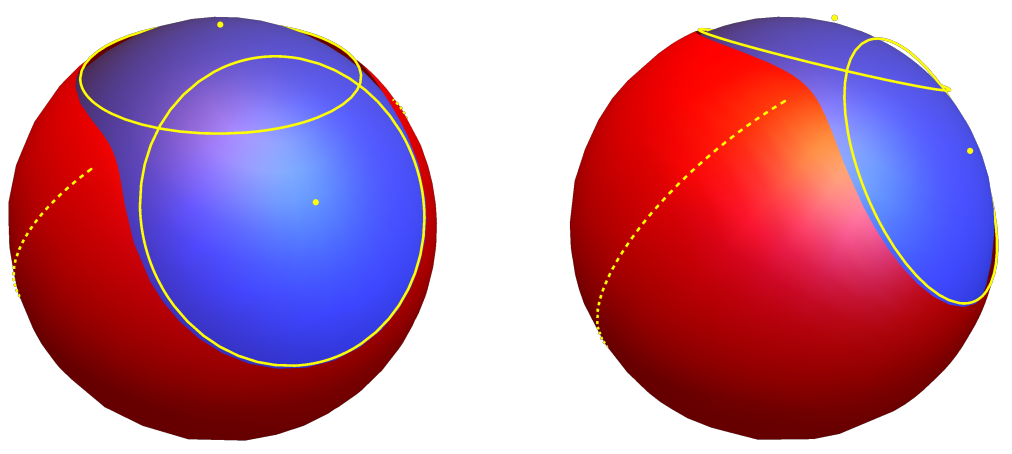

Figure 1: Picture of the droplet (red region) in case $r=1$ and $a>a_{1, c r}$. The spherical caps centered at $p_{0}$ and $p_{1}$ with geodesic radii $a /(1+2 a)$ are also represented, as well as the support of the motherbody (dashed line inside the droplet). The boundary of the droplet is mapped by stereographic projection onto an ellipse in the complex plane [11].

interval along the big circle that separates the two points $p_{0}$ and $p_{1}$. See Figure 1 that is taken from [11] and compare also with [7, Figure 4]. This situation was analyzed in [11 and it was shown that the boundary of the droplet is mapped by stereographic projection to an ellipse in the complex plane. This fact can also be deduced from earlier work by Gustafsson and Tkachev in [26, Example 3].

The approach of [11] is to first characterize the motherbody by means of an equilibrium problem from logarithmic potential theory [44, 45]. This equilibrium problems asks for the minimizer of

$$
\iint \log \frac{1}{|x-y|} d \mu(x) d \mu(y)+2 \int V(x) d \mu(x)
$$

among probability measures $\mu$ on $\mathbb{R}$, with

$$
V(x)=\frac{1+a}{2} \log \left(x^{2}+b^{-2}\right)-\frac{a}{2} \log \left(x^{2}+b^{2}\right),
$$

where $\pm i b, b>1$, are the images of the two points $p_{0}, p_{1}$ under stereographic projection onto the complex plane. The minimizer is calculated explicitly in [11, Theorem 1.6], see also [43]. The external field is only weakly admissible 
[27. 47] and for a fixed $b>1$ there is a critical value $a_{c r}$ such that the minimizer $\mu_{V}$ is compactly supported if and only if $a>a_{c r}$. Out of the Stieltjes transform of $\mu_{V}$ a meromorphic function $S$ is then constructed that is shown to be the spherical Schwarz function of a certain domain $\Omega$ in the sense that its boundary is characterized by

$$
\partial \Omega: \quad S(z)=\frac{\bar{z}}{1+|z|^{2}} .
$$

After pulling back to the sphere with inverse stereographic projection, the domain $\Omega$ is then proved to give the droplet $D_{\sigma}$ and $\mu_{V}$ gives the motherbody.

\subsection{Stereographic projection and removal of symmetry}

In this paper we extend the approach of [11] to $r+1$ points on the sphere. As in [11] we project onto the complex plane where we do all calculations. Instead of the equilibrium problem (1.6), 1.7) we study a vector equilibrium problem for a vector of $r$ measures. This will be described in section 2.1 below. In this section we first describe what we aim to achieve in the complex plane.

We move from the sphere to the complex plane by stereographic projection, where the south pole is mapped to 0 and the north pole to $\infty$. The points $p_{0}, \ldots, p_{r}$ are projected to $r+1$ points with absolute value $q^{-\frac{1}{r+1}}$ for some number $q>0$. The projected points will be the solutions of the equation $z^{r+1}+q=0$, namely

$$
p_{j} \mapsto q^{-\frac{1}{r+1}} e^{i \theta_{j}}, \quad \theta_{j}=\frac{\pi}{r+1}+\frac{2 j \pi}{r+1}, \quad \text { for } j=0,1, \ldots, r .
$$

The case $q<1$ corresponds to points $p_{j}$ in the northern hemisphere, and $q>1$ to points in the southern hemisphere.

The angles $\theta_{j}$ are chosen in such a way that the meridians separating the points $p_{0}, \ldots, p_{r}$ at equal distances are mapped to the $r+1$-star

$$
\left\{z \in \mathbb{C} \mid z^{r+1} \in[0, \infty)\right\} .
$$

The droplet $D_{\sigma}$ is mapped to a domain $\Omega \subset \mathbb{C} \cup\{\infty\}$, and $\mu_{\sigma}$ is mapped to its pushforward on $\Omega$ which takes the form

$$
d \mu_{\Omega}(z)=\left.\frac{d A(z)}{t \pi\left(1+|z|^{2}\right)^{2}}\right|_{\Omega}
$$


where $d A(z)$ is the planar Lebesgue measure on $\mathbb{C}$. and

$$
t=\frac{1}{1+\sigma\left(\mathbb{S}^{2}\right)}=\frac{1}{1+(r+1) a}
$$

The properties $(1.2)$ translate into

$$
U^{\mu_{\Omega}}(z)+a \log \frac{1}{\left|z^{r+1}+q^{-1}\right|}+\frac{1+(r+1) a}{2} \log \left(1+|z|^{2}\right) \begin{cases}=c_{1}, & z \in \Omega \\ \geq c_{1}, & z \in \mathbb{C} .\end{cases}
$$

for some constant $c_{1}$.

The motherbody $\sigma^{*}$ (that we are looking for in this paper and whose existence we do not a priori assume) satisfying (1.5) corresponds to a probability measure $\mu^{*}$ on $\left\{z \mid z^{r+1} \in[0, \infty)\right\}$ with the property that

$$
\begin{array}{ll}
U^{\mu^{*}}=U^{\mu_{\Omega}}+c_{2}, & \text { on } \mathbb{C} \backslash \Omega, \\
U^{\mu^{*}} \geq U^{\mu_{\Omega}}+c_{2}, & \text { on } \mathbb{C},
\end{array}
$$

for some other constant $c_{2}$. The aim of the paper is to construct the domain $\Omega$ and measures $\mu^{*}$ and $\mu_{\Omega}$ satisfying the conditions 1.11$)$ and $(1.12$.

The probability measures $\mu^{*}$ and $\mu_{\Omega}$ will be invariant under rotations around the origin over angle $\frac{2 \pi}{r+1}$. For our computations it will be convenient to remove the rotational symmetry, and change variables $z \mapsto z^{r+1}$. Then $\mu^{*}$ will correspond to a probability measure $\mu_{1}$ on $[0, \infty)$, and $\mu_{\Omega}$ to a probability measure $\mu_{U}$ on the set

$$
U=\left\{z^{r+1} \mid z \in \Omega\right\}
$$

and $\mu_{U}$ takes the form

$$
d \mu_{U}(z)=\left.\frac{1}{(r+1) t \pi} \frac{d A(z)}{|z|^{\frac{2 r}{r+1}}\left(1+|z|^{\frac{2}{r+1}}\right)^{2}}\right|_{U} .
$$

which comes from applying the change of variables to 1.9

Our approach will be to construct $\mu_{1}$ first as the first component of the minimizer of a vector equilibrium problem (VEP) for $r$ measures. Besides $\mu_{1}$ there will be further measures $\mu_{2}, \ldots, \mu_{r}$ that play auxiliary roles. They do not have a direct interpretation for the problem at hand, though.

In the next section we will state the VEP without trying to motivate the form that it takes. It is actually by no means obvious that this VEP 
is relevant for our problem, and it will be our main result that $\mu_{1}$ after symmetrization gives indeed a measure $\mu^{*}$ that can be identified as the image of the motherbody under stereographic projection. However, for $r=1$, the $\mathrm{VEP}$ is an equilibrium problem for one measure that, after symmetrization, can be identified with (1.7).

The VEP gives rise to an algebraic structure and this will allow us to find a domain $U$ with a measure (1.14). Through $(1.13)$ we find a domain $\Omega$ with rotational symmetry and the measure $\mu_{\Omega}$ as in $(1.9)$. We prove that it has the properties 1.11 and 1.12 .

The VEP depends on two parameters $q>0$ and $t \in(0,1)$, that ultimately will play the roles of the parameters appearing in (1.8) and (1.10), as we will show in the end.

\section{Statement of results}

\subsection{Vector equilibrium problem}

Let $r \geq 2$ be an integer, and let $q>0,0<t<1$ be real parameters. Our starting point is a vector equilibrium problem that asks to minimize the energy functional

$$
\begin{array}{rl}
\mathcal{E}\left(\mu_{1}, \mu_{2}, \ldots, \mu_{r}\right)=\sum_{j=1}^{r} & I\left(\mu_{j}\right)-\sum_{j=1}^{r-1} I\left(\mu_{j}, \mu_{j+1}\right) \\
& +\frac{1-t}{t} I\left(\mu_{1}, \delta_{-q^{-1}}\right)-\frac{r+t}{t} I\left(\mu_{r}, \delta_{(-1)^{r} q}\right),
\end{array}
$$

depending on $r$ measures. Here $\delta_{-q^{-1}}$ and $\delta_{(-1)^{r} q}$ denote Dirac point masses. As usual we write

$$
I(\mu, \nu)=\int U^{\mu} d \nu=\iint \log \frac{1}{|x-y|} d \mu(x) d \nu(y)
$$

for the mutual logarithmic energy of $\mu$ an $\nu$, and $I(\mu)=I(\mu, \mu)$ for the logarithmic energy of $\mu$.

Our aim is to minimize (2.1) over a vector of measures satisfying certain conditions. We emphasize that a measure (without any adjective) will always refer to a positive measure. We also encounter negative measures or signed measures in this paper, but in such a context the adjective will always be mentioned. 
Definition 2.1. The vector equilibrium problem (VEP) asks to minimize the energy functional 2.1) over vectors $\left(\mu_{1}, \ldots, \mu_{r}\right)$ of measures subject to the conditions

(a) $\operatorname{supp}\left(\mu_{j}\right) \subset \Delta_{j}$ for every $j$, where

$$
\Delta_{j}= \begin{cases}{[0, \infty),} & \text { if } j \text { is odd } \\ (-\infty, 0], & \text { if } j \text { is even, }\end{cases}
$$

(b) the total mass of $\mu_{j}$ is

$$
\mu_{j}\left(\Delta_{j}\right)=1+\frac{j-1}{t}, \quad \text { for } j=1, \ldots, r .
$$

Throughout the paper we will write

$$
\mu_{0}=\left(1-\frac{1}{t}\right) \delta_{-q^{-1}}, \quad \mu_{r+1}=\left(1+\frac{r}{t}\right) \delta_{(-1)^{r} q} .
$$

Then (2.3) is also satisfied for $j \in\{0, r+1\}$, but note that $\mu_{0}$ is a negative measure (since $0<t<1$ ). Moreover, 2.1) takes the compact form

$$
\mathcal{E}\left(\mu_{1}, \ldots, \mu_{r}\right)=\sum_{j=1}^{r} I\left(\mu_{j}\right)-\sum_{j=0}^{r} I\left(\mu_{j}, \mu_{j+1}\right),
$$

that includes $\mu_{0}$ and $\mu_{r+1}$ as well, but $\mu_{0}$ and $\mu_{r+1}$ remain fixed in the VEP.

Vector equilibrium problems were first introduced by Gonchar and Rakhmanov in their study of Hermite-Padé approximation [21, 22], see also [42]. They also appear in ensembles of random matrices that are related to multiple orthogonal polynomials, see [2, 28] and references cited therein.

The energy functional 2.5 involves an attraction between neighboring measures that is of Nikishin type, and this has appeared in a number of situations before. What is special is that the total masses $(2.3)$ are in an arithmetic progression that is increasing with steps $1 / t$. It is more common that the masses are in an arithmetic progression that decreases from 1 to 0 see e.g. [17] and the examples in [2, 28].

The VEP of Definition 2.1 is weakly admissible in the sense of [27] as we show next.

Lemma 2.2. The vector equilibrium problem is weakly admissible. There is a unique minimizer, denoted $\left(\mu_{1}, \ldots, \mu_{r}\right)$. The measures $\mu_{2}, \ldots, \mu_{r}$ have full supports

$$
\operatorname{supp}\left(\mu_{j}\right)=\Delta_{j}=(-1)^{j-1}[0, \infty), \quad \text { for } j=2, \ldots, r .
$$


Proof. To check the conditions in Assumption 2.1 of [27], we write the energy functional (2.5) in the form

$$
\sum_{1 \leq i, j \leq r} c_{i j} I\left(\mu_{i}, \mu_{j}\right)+\sum_{j=1}^{r} \int V_{j} d \mu_{j}
$$

with

$$
c_{i j}= \begin{cases}1 & \text { if } i=j \\ -\frac{1}{2} & \text { if }|i-j|=1 \\ 0 & \text { otherwise }\end{cases}
$$

and

$$
V_{j}(x)= \begin{cases}-U^{\mu_{0}}(x)=\left(1-\frac{1}{t}\right) \log \left|x+q^{-1}\right|, & \text { if } j=1, \\ -U^{\mu_{r+1}}(x)=\left(1+\frac{r}{t}\right) \log \left|x-(-1)^{r} q\right|, & \text { if } j=r \\ \equiv 0, & \text { otherwise }\end{cases}
$$

The interaction matrix $C=\left(c_{i j}\right)$ is symmetric and positive definite, and each $V_{j}$ is continuous on $\Delta_{j}$, since $-q^{-1} \notin \Delta_{1}$ and $(-1)^{r} q \notin \Delta_{r}$.

The prescribed total masses $m_{j}=\mu_{j}\left(\Delta_{j}\right)$ from (2.3) come in an arithmetic progression which implies by (2.7) that

$$
\sum_{j=1}^{r} c_{i j} m_{j}=0, \quad \text { for } i=2, \ldots, r-1,
$$

and also

$$
\sum_{j=1}^{r} c_{i j} m_{j}= \begin{cases}\frac{1}{2} m_{0}=\frac{1}{2}\left(1-\frac{1}{t}\right), & \text { for } i=1, \\ \frac{1}{2} m_{r+1}=\frac{1}{2}\left(1+\frac{r}{t}\right), & \text { for } i=r .\end{cases}
$$

It follows from (2.8), (2.9) and 2.10$)$ that, for every $i=1, \ldots, r$,

$$
V_{i}(x)-\left(\sum_{j=1}^{r} c_{i, j} \mu_{j}\left(\Delta_{j}\right)\right) \log \left(1+|x|^{2}\right) \rightarrow 0, \quad \text { as } x \in \Delta_{j} \rightarrow \pm \infty .
$$

Thus all conditions of Assumption 2.1 in [27] are satisfied, and the VEP is weakly admissible. Then there is a unique minimizer by [27, Corollary 2.7].

Given the other measures, the problem for $\mu_{j}$ (for $1 \leq j \leq r$ ) is to minimize

$$
I\left(\mu_{j}\right)-I\left(\mu_{j}, \mu_{j-1}+\mu_{j+1}\right)
$$


among measures on $\Delta_{j}$ with total mass 2.3 . Since $\mu_{j-1}+\mu_{j+1}$ is a positive measure for $j \geq 2$, it follows that $\mu_{j}$ is a balayage measure (see [45] for the notion of balayage)

$$
\mu_{j}=\frac{1}{2} \operatorname{Bal}\left(\mu_{j-1}+\mu_{j+1}, \Delta_{j}\right) \quad \text { for } j=2, \ldots, r,
$$

and $\mu_{j}$ has full support for $j \geq 2$, see also 3.2 for the expression of the density of the balayage of a measure on $(-\infty, 0]$ onto $[0, \infty)$. There is a similar formula for the balayage of a measure on $[0, \infty)$ to $(-\infty, 0]$ that shows that it has indeed a full support.

The balayage property 2.11 means that

$$
2 U^{\mu_{j}}=U^{\mu_{j-1}}+U^{\mu_{j+1}} \quad \text { on } \Delta_{j}, \quad \text { for } j=2, \ldots, r,
$$

and this will be important for us in what follows.

The measure $\mu_{1}$ is the main player in the game. The argument in the proof of Lemma 2.2 leading to (2.11) does not work for $j=1$, since $\mu_{0}$ is a negative measure. Therefore the balayage of $\mu_{0}+\mu_{2}$ onto $\Delta_{1}=[0, \infty)$ is not necessarily positive on the full half-line. However, if it is positive then (2.11) and 2.12 hold for $j=1$ as well, and then also $\mu_{1}$ has a full support. It turns out that this happens for $t$ sufficiently large (i.e., sufficiently close to 1$)$.

Our first main result is about the structure of the support $\Sigma_{1}=\operatorname{supp}\left(\mu_{1}\right)$ of $\mu_{1}$. There are four possible cases that will be indicated with acronyms BIS $=$ Bounded Interval Support, UIS = Unbounded Interval Support, TIS $=$ Two Interval Support, and FIS $=$ Full Interval Support.

In situations where we want to emphasize the dependence on $t$ of the various notions that we introduced (and of others that are still to come), we append a subscript $t$. Hence we write for example $\mu_{1, t}, \Sigma_{1, t}$, and so on.

Theorem 2.3. Fix $q>0$. Let $\left(\mu_{1}, \ldots, \mu_{r}\right)$ be the minimizer of the vector equilibrium problem depending on the parameter $t \in(0,1)$.

(a) There are four possible cases for $\Sigma_{1}=\operatorname{supp}\left(\mu_{1}\right)$, depending on $t$, namely there exist $0<x_{1}<x_{2}<\infty$ such that either BIS : $\Sigma_{1}=$ $\left[0, x_{1}\right]$, or $\boldsymbol{U I S}: \Sigma_{1}=\left[x_{2}, \infty\right)$, or $\boldsymbol{T I S}: \Sigma_{1}=\left[0, x_{1}\right] \cup\left[x_{2}, \infty\right)$, or $\boldsymbol{F I S}: \Sigma_{1}=[0, \infty)$.

(b) For each $j=1, \ldots, r$ the measure $t \mu_{j, t}$ increases as a function of $t \in(0,1)$. 
(c) Suppose $0<q<1$. Then 0 is always in the support of $\mu_{1}$ (and so UIS case does not occur for any $t \in(0,1))$.

(d) The measure $\mu_{1}$ has a density that is real analytic on the interior of its support with a square-root vanishing at $x_{1}$ in the BIS and $\boldsymbol{T I S}$ cases, and at $x_{2}$ in the $\boldsymbol{U I S}$ and $\boldsymbol{T I S}$ cases.

(e) There exist constants $c_{0}>0$ and $c_{\infty}>0$ such that

$$
\frac{d \mu_{1}(x)}{d x}=c_{0} x^{-\frac{r}{r+1}}\left(1+O\left(x^{\frac{1}{r+1}}\right)\right) \quad \text { as } x \rightarrow 0+
$$

in $\boldsymbol{B I S}, \boldsymbol{T I S}$ and $\boldsymbol{F I S}$ cases, and

$$
\frac{d \mu_{1}(x)}{d x}=c_{\infty} x^{-\frac{r+2}{r+1}}\left(1+O\left(x^{-\frac{1}{r+1}}\right)\right) \quad \text { as } x \rightarrow \infty
$$

in $\boldsymbol{U I S}, \boldsymbol{T I S}$ and $\boldsymbol{F I S}$ cases.

The proof of Theorem 2.3 is in section 3, except for the proof of part (e) which is in section 4.1.2.

It follows from Theorem 2.3 that for $0<q<1$, there are two critical values

$$
0 \leq t_{1, c r} \leq t_{2, c r} \leq 1,
$$

depending on $q$, such that we are in BIS case for $0<t \leq t_{1, c r}$, in the TIS case for $t_{1, c r}<t<t_{2, c r}$, and in FIS case for $t_{2, c r} \leq t<1$. For $r \geq 2$, the inequalities in (2.15) are actually strict inequalities and each of the three possible cases occurs for some values of $t$.

Ultimately, $t$ will be related by equation (1.10) to the strength $a$ of the fixed charges on the sphere. The critical values $t_{1, c r}$ and $t_{2, c r}$ will correspond to $a_{2, c r}$ and $a_{1, c r}$ (in that order) that are used at the end of section 1.1 .

Remark 2.4. There is a symmetry between $q$ and $q^{-1}$ that allows us to restrict attention to $0<q<1$.

Let $\vec{\mu}=\left(\mu_{1}, \ldots, \mu_{r}\right)$ be a vector of measures as in the VEP of Definition 2.1. Let $\nu_{j}$ be the image of $\mu_{j}$ under the inversion $x \mapsto 1 / x$, i.e., $\nu_{j}$ is the measure on $\Delta_{j}$ with

$$
\int f d \nu_{j}=\int f\left(\frac{1}{x}\right) d \mu_{j}(x)
$$

for a function $f$ on $\Delta_{j}$. Then it is an easy calculation to show that

$$
I\left(\nu_{j}, \nu_{k}\right)=I\left(\mu_{j}, \mu_{k}\right)+\int \log |x| d \mu_{j}(x) \int d \mu_{k}+\int \log |x| d \mu_{k}(x) \int d \mu_{j} .
$$




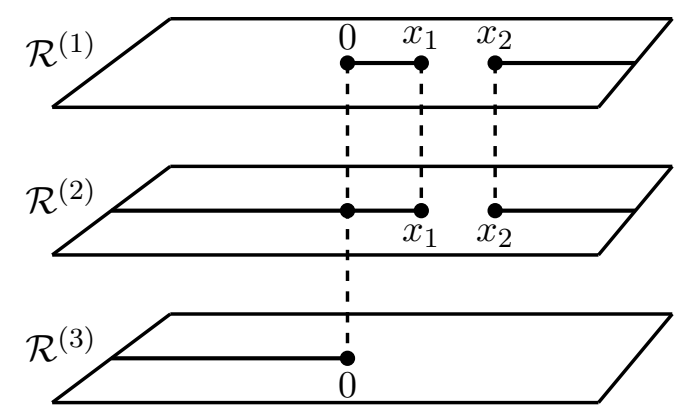

Figure 2: The Riemann surface $\mathcal{R}$ in TIS case (for $r=2$ )

Let us use $\mathcal{E}_{q}$ to denote the energy functional 2.1 corresponding to the parameter $q>0$. Then using (2.17) and the total masses $(2.3)$ of the measures we find after straightforward calculations that

$$
\mathcal{E}_{1 / q}(\vec{\nu})=\mathcal{E}_{q}(\vec{\mu})-\frac{r(r+2 t-1)}{t^{2}} \log q .
$$

Thus whenever $\vec{\mu}=\left(\mu_{1}, \ldots, \mu_{r}\right)$ is the minimizer of the VEP with parameter $q$, then $\vec{\nu}=\left(\nu_{1}, \ldots, \nu_{r}\right)$ is the minimizer with parameter $1 / q$.

Due to this symmetry between $q$ and $q^{-1}$, the support of $\mu_{1}$ is always unbounded for $q>1$, since for $0<q<1$ the support contains 0 by part (c) of Theorem 2.3. Instead of the BIS case, we then have the UIS case for $t$ up to the first critical value. It is then continued with the TIS case, and after a second critical value with the FIS case.

The above also shows that for $q=1$, the measure $\mu_{1}$ is invariant under the inversion $x \mapsto 1 / x$. Then we do not have a BIS or UIS case, but we start with a TIS case for $t$ up to a critical value, followed by the FIS case.

\subsection{A meromorphic function on a Riemann surface}

In what follows we restrict to the case $0<q<1$. Then $0 \in \operatorname{supp}\left(\mu_{1}\right)$ by Theorem 2.3 (c) and we are in one of the BIS, TIS or FIS cases. We also let $0<t<1$. Our further results are based on the consideration of a Riemann surface.

Definition 2.5. The Riemann surface $\mathcal{R}$ (see Figure 2) has $r+1$ sheets 
$\mathcal{R}^{(j)}, j=1, \ldots, r+1$, where

$$
\begin{aligned}
\mathcal{R}^{(1)} & =\mathbb{C} \backslash \operatorname{supp}\left(\mu_{1}\right), \\
\mathcal{R}^{(j)} & =\mathbb{C} \backslash\left(\operatorname{supp}\left(\mu_{j-1}\right) \cup \operatorname{supp}\left(\mu_{j}\right)\right), \quad \text { for } j=2, \ldots, r+1, \\
\mathcal{R}^{(r+1)} & =\mathbb{C} \backslash \operatorname{supp}\left(\mu_{r}\right)
\end{aligned}
$$

where $\left(\mu_{1}, \ldots, \mu_{r}\right)$ is the unique minimizer for the VEP of Definition 2.1, and we recall that $\operatorname{supp}\left(\mu_{1}\right)=\Sigma_{1} \subset[0, \infty)$ and $\operatorname{supp}\left(\mu_{j}\right)=\Delta_{j}=(-1)^{j-1}[0, \infty)$ for $j=2, \ldots, r$. Sheet $\mathcal{R}^{(j)}$ is connected to sheet $\mathcal{R}^{(j+1)}$ along the support of $\mu_{j}$ in the usual crosswise manner for $j=1, \ldots, r$. We also add two (in BIS case) or one (in other cases) points at infinity in order to obtain a compact Riemann surface $\mathcal{R}$.

A count of branch points, together with the Riemann-Hurwitz formula, see e.g. [46], shows that $\mathcal{R}$ has genus zero in the BIS and FIS cases, while the genus is one in the TIS case.

The Stieltjes transform of the measure $\mu_{j}$ is

$$
F_{j}(z)=\int \frac{d \mu_{j}(x)}{z-x}, \quad z \in \mathbb{C} \backslash \operatorname{supp}\left(\mu_{j}\right)
$$

This is also defined for $j=0$ and $j=r+1$ in which cases we have the simple rational functions

$$
F_{0}(z)=\frac{-1+t}{t\left(z+q^{-1}\right)}, \quad F_{r+1}(z)=\frac{r+t}{t\left(z-(-1)^{r} q\right)}
$$

We use the Stieltjes transforms to define a function on $\mathcal{R}$.

Definition 2.6. The function $\Phi$ is defined on the Riemann surface via its restrictions $\Phi^{(j)}, j=1, \ldots, r+1$, to the various sheets, by

$$
\Phi^{(j)}(z)=t F_{j}(z)-t F_{j-1}(z), \quad z \in \mathcal{R}^{(j)},
$$

for $j=1, \ldots, r+1$.

Differentiating the identity 2.12 we obtain

$$
F_{j,+}-F_{j,-}=F_{j-1}+F_{j+1} \quad \text { on } \Delta_{j}=\operatorname{supp}\left(\mu_{j}\right), \quad \text { for } j=2, \ldots, r,
$$

which means in view of 2.21 that $\Phi_{ \pm}^{(j)}=\Phi_{\mp}^{(j+1)}$ on $\operatorname{supp}\left(\mu_{j}\right)$ for $j=$ $2, \ldots, r$. Thus $\Phi$ is analytic across the cut connecting sheets $\mathcal{R}^{(j)}$ and $\mathcal{R}^{(j+1)}$ 
for $j \geq 2$. $\Phi$ is also analytic across the cut connecting sheets $\mathcal{R}^{(1)}$ and $\mathcal{R}^{(2)}$, as this follows from the variational condition associated with the VEP

$$
2 U^{\mu_{1}}=U^{\mu_{0}}+U^{\mu_{2}}+c \quad \text { on } \operatorname{supp}\left(\mu_{1}\right),
$$

which upon differentation leads to 2.22 on $\operatorname{supp}\left(\mu_{j}\right)$ for $j=1$ as well. Thus $\Phi$ is meromorphic on $\mathcal{R}$ and it has a number of crucial properties that will be discussed in section 4.1 .

\subsection{The subset $U$}

With the help of $\Phi$ we define a subset $U$ of the complex plane that will lead to the droplet.

Definition 2.7. The set $U \subset \mathbb{C} \cup\{\infty\}$ is defined by

$$
U=\overline{\left\{z \in \mathbb{C} \mid(\operatorname{Im} z) \cdot \operatorname{Im}\left(z \Phi^{(1)}(z)\right)<0\right\}}
$$

We write $U_{t}$ if we want to emphasize the dependence of $U$ on the parameter $0<t<1$.

Theorem 2.8. Let $0<q<1$ be fixed. Then the following hold.

(a) $U$ is a closed set with the properties $\Sigma_{1} \subset U$ and $-q^{-1}$.

(b) For $0<t \leq t_{1, c r}$ (the BIS case), $U$ is a bounded simply connected set.

(c) For $t_{1, c r}<t<t_{2, c r}$ (the $\boldsymbol{T I S}$ case), $U$ consists of two disjoint components: a bounded component containing $\left[0, x_{1}\right]$ and an unbounded component containing $\left[x_{2}, \infty\right)$. The complement $\mathbb{C} \backslash U$ is a bounded doubly connected domain.

(d) For $t_{2, c r} \leq t<1$ (the $\boldsymbol{F I S}$ case), $U$ is unbounded and connected. The complement $\mathbb{C} \backslash U$ is bounded and simply connected.

(e) $t \mapsto U_{t}$ is increasing with $t$.

(f) $z \Phi^{(1)}(z)$ is real-valued on the boundary $\partial U$ and

$$
z \Phi^{(1)}(z)=\frac{|z|^{\frac{2}{r+1}}}{1+|z|^{\frac{2}{r+1}}} \quad \text { for } z \in \partial U .
$$

The proof of Theorem 2.8 is in section 4. See Figure 3 for plots of $U$ in the three cases. 

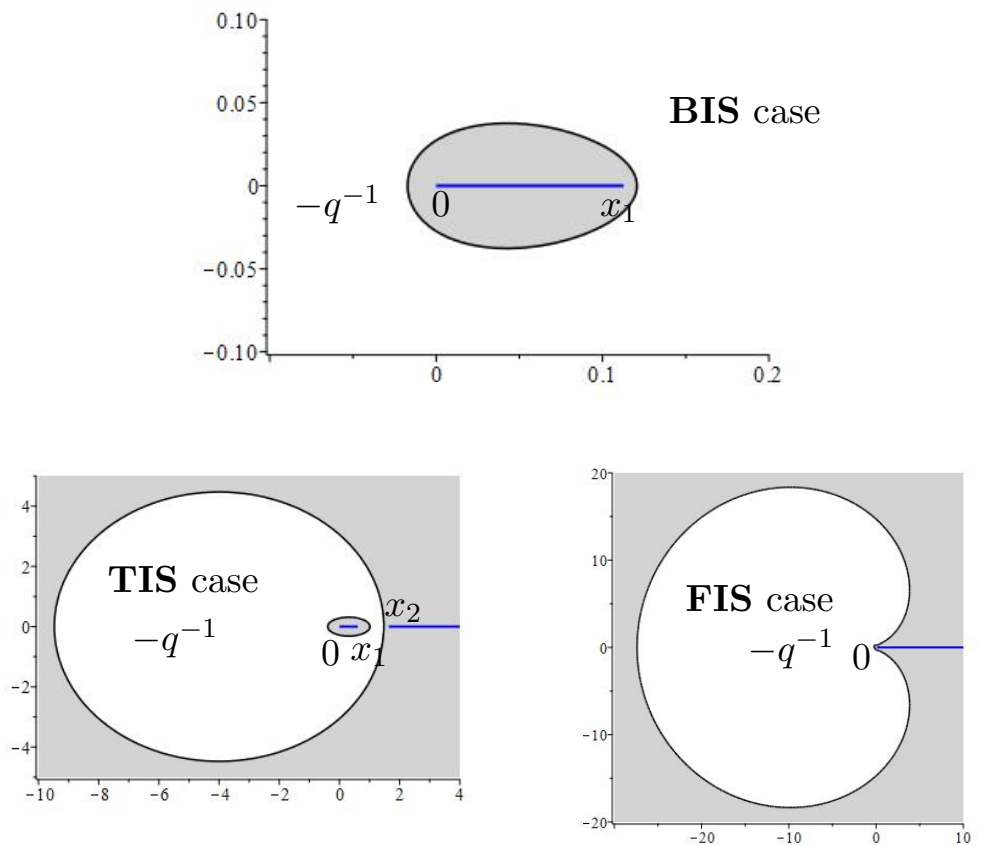

Figure 3: Domain $U$ (shaded region) in the three cases. The blue line denotes the support of $\mu_{1}$ and it is located within $U$, while $-q^{-1}$ is located outside $U$.

\subsection{The symmmetric domain $\Omega$ with spherical measure}

We now introduce $r+1$ fold symmetry.

Definition 2.9. We define a domain $\Omega$ (see Figure 4)

$$
\Omega=\left\{z \in \mathbb{C} \mid z^{r+1} \in U\right\},
$$

and a function

$$
S(z)=z^{r} \Phi^{(1)}\left(z^{r+1}\right)
$$

which we call the spherical Schwarz function of $\partial \Omega$.

We call $S$ the spherical Schwarz function because of the property

$$
S(z)=\frac{\bar{z}}{1+|z|^{2}} \quad \text { for } z \in \partial \Omega
$$



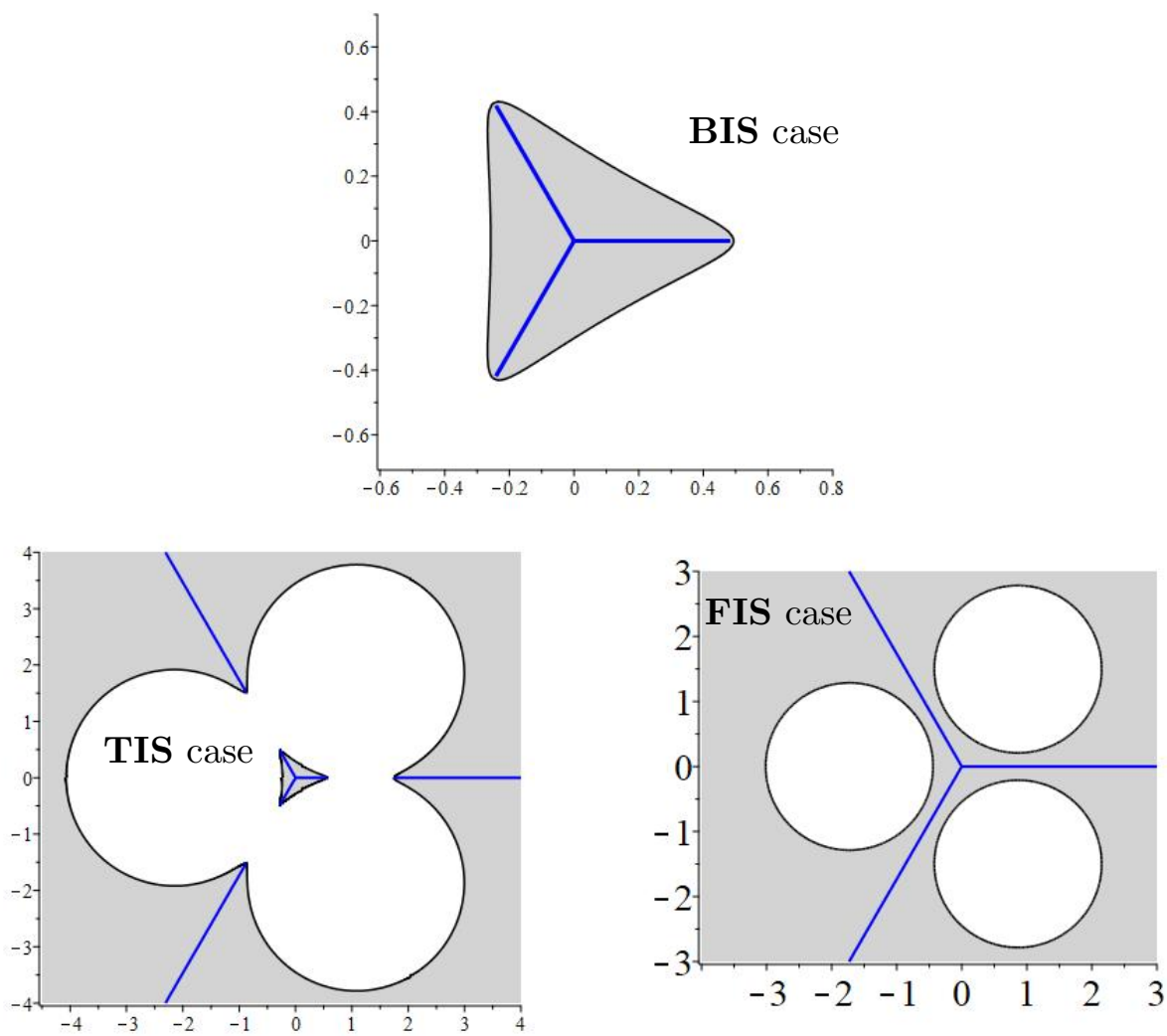

Figure 4: Domain $\Omega$ (shaded region) in the three cases for $r=2$. The blue lines are the support of $\mu^{*}$.

which follows from (2.24) and the definitions in Definition 2.9. It readily follows from 2.27 that $\frac{S(z)}{1-z S(z)}=\bar{z}$ for $z \in \partial \Omega$, and so $\frac{S(z)}{1-z S(z)}$ is the usual Schwarz function of $\partial \Omega$, and the two notions are very much intertwined, see also the paper [13] on vertex dynamics on the sphere.

Then $S$ is defined and meromorphic on $\left\{z \in \mathbb{C} \mid z^{r+1} \notin \operatorname{supp}\left(\mu_{1}\right)\right\}$ with poles at the solutions of $z^{r+1}=-q^{-1}$, with the behavior $z S(z) \rightarrow 1$ as $z \rightarrow \infty$. Also $S$ has an analytic continuation to a meromorphic function on a compact $r+1$ sheeted Riemann surface where $S(z)=z^{r} \Phi^{(j)}\left(z^{r+1}\right)$ on the $j$ th sheet. This analytic continuation has poles on the $(r+1)$ st sheet given by the solutions of $z^{r+1}=(-1)^{r} q$.

We next define the two measures $\mu_{\Omega}$ and $\mu^{*}$. 
Definition 2.10. We define a measure $\mu_{\Omega}$ on $\Omega$ by

$$
d \mu_{\Omega}(z)=\left.\frac{1}{\pi t} \frac{d A(z)}{\left(1+|z|^{2}\right)^{2}}\right|_{\Omega}
$$

and $\mu^{*}$ as the unique measure on the $r+1$ star

$$
\left\{z \mid z^{r+1} \in \mathbb{R}^{+}\right\}
$$

that is invariant under rotation $z \mapsto e^{\frac{2 \pi i}{r+1}} z$ and whose pushforward under $z \mapsto z^{r+1}$ is equal to $\mu_{1}$ (the first component of the minimizer of the VEP).

This leads to the final main result of the paper.

Theorem 2.11. (a) $\mu_{\Omega}$ is a probability measure on $\Omega$, and $\mu^{*}$ is a probability measure on 2.29.

(b) $t \mapsto \Omega_{t}, t \mapsto t \mu_{\Omega, t}$ and $t \mapsto t \mu_{t}^{*}$ are increasing for $t \in(0,1)$.

(c) There is a constant $c_{1}=c_{1, t}$ such that

$$
U^{\mu_{\Omega}}(z)-\frac{1-t}{(r+1) t} \log \left|z^{r+1}+q^{-1}\right|+\frac{1}{2 t} \log \left(1+|z|^{2}\right) \begin{cases}=c_{1}, & z \in \Omega \\ \geq c_{1}, & z \in \mathbb{C} .\end{cases}
$$

If $\Omega$ is unbounded, then $c_{1}=0$.

(d) There is a constant $c_{2}=c_{2, t}$ such that

$$
U^{\mu_{\Omega}}(z)-U^{\mu^{*}}(z)\left\{\begin{array}{l}
=c_{2}, \quad z \in \mathbb{C} \backslash \Omega, \\
\leq c_{2}, \quad z \in \mathbb{C} .
\end{array}\right.
$$

If $\Omega$ is bounded, then $c_{2}=0$.

The proof of Theorem 2.11 is in section 5

Parts (c) and (d) of Theorem 2.11 tell us that the equations (1.11) and (1.12) are satisfied provided

$$
a=\frac{1-t}{(r+1) t}
$$

which agrees with 1.10 . Thus, as already explained, Theorem 2.11 shows that the image of $\Omega$ under inverse stereographic projection is the droplet $D_{\sigma}$ on the unit sphere, and the pullback of $\mu^{*}$ is the motherbody $\sigma^{*}$. 
Remark 2.12. The domain $\Omega_{t}$ increases with $t$ according to part (b) of Theorem 2.11. It is an instance of Laplacian growth (or Hele-Shaw flow) in the spherical metric. We refer to [25] and the references therein for more on the interesting topic of Laplacian growth and its many connections.

Figure 4 contains a plot of $\Omega$ in the various cases for the value $r=2$. It is interesting to note that in the BIS case $\Omega$ coincides with the droplet in the normal matrix model with a cubic potential, see e.g. [4, 18, 48. The eigenvalues in this random matrix model tend to the droplet with a uniform density (in contrast to 2.28 which is uniform in the spherical metric), and the zeros of related orthogonal polynomials tend to the motherbody. The limiting zero counting measure is characterized by a vector equilibrium problem in [4] that is however different from the VEP of Definition 2.1, see also [31] for the case $r \geq 3$. Our VEP can be seen as a spherical analogue from the VEPs in [4, 31].

In the normal matrix model with a cubic potential the droplet grows up to a critical time and then cusps appear on the boundary of the droplet that cause a breakdown of the model, (see however [33, 35, 36, 37] for continuations beyond breakdown). In our model there is no breakdown since the transition to the TIS takes place before we reach the cusp situation.

\section{Proof of Theorem 2.3}

\subsection{Proof of part (a)}

\subsubsection{A more general result}

Given the second component $\mu_{2}$ of the solution of the VEP of Definition 2.1. $\mu_{1}$ is the probability measure $\mu$ on $[0, \infty)$ that minimizes $I(\mu)-I(\mu, \sigma)$ where $\sigma=\mu_{0}+\mu_{2}$ is a signed measure with integral $\int \sigma=2$. Part (a) of Theorem 2.3 will follow from the following more general result, where it is important that the negative part of $\sigma$ is a Dirac point mass. Note that $\sigma$ used in this section is not related to $\sigma$ from (1.1).

Proposition 3.1. Suppose $\sigma=-A \delta_{-q^{-1}}+\sigma^{+}$where $A>0$ and $\sigma^{+}$is a measure on $(-\infty, 0]$ with $A<\int d \sigma^{+}<\infty$. Then there is a unique $\mu$ on $[0, \infty)$ that minimizes

$$
I(\mu)-I(\mu, \sigma)
$$

among all measures on $[0, \infty)$ with $\int d \mu=\frac{1}{2} \int \sigma$. The support $\Sigma=\operatorname{supp}(\mu)$ takes one of the forms described in Theorem 2.3 (a), namely $\Sigma$ is a bounded interval $\left[0, x_{1}\right]$ containing 0 , an unbounded interval $\left[x_{2}, \infty\right)$ not containing 
0 , the disjoint union of two intervals $\left[0, x_{1}\right] \cup\left[x_{2}, \infty\right)$, or the full half-line $[0, \infty)$.

The minimization problem in Proposition 3.1 is again weakly admissible, and there is a unique minimizer $\mu$. If we relax the condition that $\mu$ is a measure and also allow signed measures, then the minimizer is the balayage

$$
\nu=\frac{1}{2} \operatorname{Bal}(\sigma,[0, \infty))
$$

which is known to have the density

$$
\frac{d \nu}{d x}=\frac{1}{2 \pi \sqrt{x}} \int_{-\infty}^{0} \frac{\sqrt{|s|}}{x-s} d \sigma(s), \quad 0<x<\infty .
$$

If the density (3.2) happens to be non-negative on $[0, \infty)$, then $\mu=\nu$ solves the minimization problem and $\operatorname{supp} \mu=[0, \infty)$.

If $\nu$ is not a positive measure, then we use the idea of iterated balayage [15, 30]. This method is based on the fact that $\mu \leq \nu^{+}$where $\nu^{+}$is the positive part of $\nu$ in its Jordan decomposition

$$
\nu=\nu^{+}-\nu^{-} \text {. }
$$

In particular $\operatorname{supp}(\mu) \subset \operatorname{supp}\left(\nu^{+}\right)$, see [30, Lemma 3]. With this information we can restrict the minimization problem to measures supported on $\operatorname{supp}\left(\nu^{+}\right)$, and if we also allow signed measures then the minimum is attained by

$$
\nu^{+}-\operatorname{Bal}\left(\nu^{-}, \operatorname{supp}\left(\nu^{+}\right)\right)
$$

If this happens to be a positive measure then it is equal to $\mu$, and we can stop. Otherwise we repeat the above step, which leads to the following iterative procedure.

We put $\nu_{1}=\nu$, and iteratively for $k=1,2, \ldots$, we write $\nu_{k}=\nu_{k}^{+}-\nu_{k}^{-}$ where $\nu_{k}^{+}$and $\nu_{k}^{-}$are the positive and negative parts of $\nu_{k}$, and we define

$$
\nu_{k+1}=\nu_{k}^{+}-\operatorname{Bal}\left(\nu_{k}^{-}, \operatorname{supp}\left(\nu_{k}^{+}\right)\right) .
$$

The convergence properties of the sequence $\left(\nu_{k}\right)_{k}$ are not fully understood, but in cases where we can control the supports of the measures we will have that $\nu_{k}^{-} \rightarrow 0$ and $\nu_{k}^{+} \rightarrow \mu$ as $k \rightarrow \infty$.

Under the conditions of Proposition 3.1 we can indeed control the supports, and we will show that for each $k$ the support of $\nu_{k}^{+}$takes one of the forms stated in the proposition, namely $\operatorname{supp}\left(\nu_{k}^{+}\right)$is either a bounded 
interval $\left[0, x_{1, k}\right]$, an unbounded interval $\left[x_{2, k}, \infty\right)$, a union of two intervals $\left[0, x_{1, k}\right] \cup\left[x_{2, k}, \infty\right)$, or the full half-line $[0, \infty)$. Since the supports are decreasing if $k$ increases, the two sequences $\left(x_{1, k}\right)$ and $\left(x_{2, k}\right)$ are either finite (maybe even empty), or else they monotonically converge to limits $x_{1}$ and/or $x_{2}$. In this way we will be able to show that $\operatorname{supp}(\mu)$ has one of the forms in the proposition. form.

In the first step we show that the support of $\nu^{+}=\nu_{1}^{+}$has the required

\subsubsection{First step: The support of $\nu^{+}$}

For $\sigma$ as in the statement of Proposition 3.1, the density (3.2) of $\nu$ takes the form

$$
\frac{v(x)}{2 \pi \sqrt{x}}, \quad \text { with } \quad v(x)=\int_{0}^{\infty} \frac{d \rho(s)}{x+s}-\frac{A_{1}}{x+q^{-1}}, \quad 0<x<\infty,
$$

where we put $A_{1}=\frac{A}{\sqrt{q}}>0$ and $d \rho(s)=\sqrt{s} d \sigma^{+}(-s)$.

Lemma 3.2. In the above setting the following hold.

(a) $v$ has at most two zeros in $(0, \infty)$.

(b) There exist $0 \leq x_{1} \leq x_{2} \leq \infty$ such that

$$
\operatorname{supp}\left(\nu^{-}\right)=\left[x_{1}, x_{2}\right] \quad \text { and } \quad \operatorname{supp}\left(\nu^{+}\right)=\overline{\left(0, x_{1}\right) \cup\left(x_{2}, \infty\right)} .
$$

Proof. (a) Suppose, to get a contradiction, that $0<x_{0}<x_{1}<x_{2}<\infty$ are three zeros of $v$. Let $s_{1}=q^{-1}$ and write $v=f_{0}-f_{1}+f_{2}$ with

$$
f_{0}(x)=\int_{0}^{s_{1}} \frac{d \rho(s)}{x+s}, \quad f_{1}(x)=\frac{A}{x+s_{1}}, \quad f_{2}(x)=\int_{s_{1}}^{\infty} \frac{d \rho(s)}{x+s} .
$$

Then by (3.5) and the multilinearity of the determinant

$$
\operatorname{det}\left[f_{k}\left(x_{j}\right)\right]_{j, k=0}^{2}=A_{1} \int_{0}^{s_{1}} d \rho\left(s_{0}\right) \int_{s_{1}}^{\infty} d \rho\left(s_{2}\right) \operatorname{det}\left[\frac{1}{x_{j}+s_{k}}\right]_{j, k=0}^{2} .
$$

There is an explicit formula for the determinant (Cauchy determinant)

$$
\operatorname{det}\left[\frac{1}{x_{j}+s_{k}}\right]_{j, k=0}^{2}=\frac{\prod_{0 \leq j<k \leq 2}\left(x_{k}-x_{j}\right) \prod_{0 \leq j<k \leq 2}\left(s_{k}-s_{j}\right)}{\prod_{j=0}^{2} \prod_{k=0}^{2}\left(x_{j}+s_{k}\right)} .
$$


In the integral in $(3.6)$ we have $0<s_{0}<s_{1}<s_{2}$, and since also $0<x_{0}<$ $x_{1}<x_{2}$, we see that the Cauchy determinant is $>0$. Since $A_{1}>0$ it follows from (3.6) that $\operatorname{det}\left[f_{k}\left(x_{j}\right)\right]>0$ and the matrix $\left[f_{k}\left(x_{j}\right)\right]_{j, k=0}^{2}$ is invertible.

However, since $v=f_{0}-f_{1}+f_{2}$ by (3.4) and (3.5), and since $v\left(x_{j}\right)=0$ for $j=0,1,2$, it follows that

$$
\left(\begin{array}{lll}
f_{0}\left(x_{0}\right) & f_{1}\left(x_{0}\right) & f_{2}\left(x_{0}\right) \\
f_{0}\left(x_{1}\right) & f_{1}\left(x_{1}\right) & f_{2}\left(x_{1}\right) \\
f_{0}\left(x_{2}\right) & f_{1}\left(x_{2}\right) & f_{2}\left(x_{2}\right)
\end{array}\right)\left(\begin{array}{c}
1 \\
-1 \\
1
\end{array}\right)=\left(\begin{array}{l}
v\left(x_{0}\right) \\
v\left(x_{1}\right) \\
v\left(x_{2}\right)
\end{array}\right)=\left(\begin{array}{l}
0 \\
0 \\
0
\end{array}\right)
$$

and this is a contradiction, since the matrix is invertible.

(b) From part (a) we know that $v$ has at most two zeros in $(0, \infty)$. By continuity, $v$ can also have at most two sign changes in $(0, \infty)$.

If $v$ has no sign changes then $v \geq 0$ on $(0, \infty)$, since due to the fact that $\sigma$ has the density (3.4) and

$$
\int d \sigma=\int_{0}^{\infty} \frac{v(x)}{2 \pi \sqrt{x}} d x>0
$$

it cannot be fully $\leq 0$. Then we take $x_{2}=x_{1}$ in the lemma.

If $v$ has one sign change, say at $x^{*}>0$, and if there is no other zero of $v$, then $v$ is either $>0$ on $\left(0, x^{*}\right)$ and $<0$ on $\left(x^{*}, \infty\right)$, or vice versa. In the former case we take $x_{1}=x^{*}$ and $x_{2}=\infty$ and in the latter case we take $x_{1}=0$ and $x_{2}=x^{*}$. If there is another zero in $(0, \infty)$, then the inequality is not strict at this one zero, but we still take $x_{1}$ and $x_{2}$ as above, and the conclusion of part (b) holds true if $v$ has one sign change. [It is actually not possible that there is another zero, but we do not need this fact.]

If $v$ has two sign changes, say at $0<x_{1}<x_{2}<\infty$, then $v$ is either positive on $\left(0, x_{1}\right)$, negative on $\left(x_{1}, x_{2}\right)$, and positive again on $\left(x_{2}, \infty\right)$, or the other way around negative on $\left(0, x_{1}\right)$, positive on $\left(x_{1}, x_{2}\right)$ and negative on $\left(x_{2}, \infty\right)$. [Now we can be sure that the inequalities are strict since there are no more than two zeros by part (a).] The latter possibility cannot happen, which we can see by adding $\epsilon \delta_{-\epsilon^{2}}$ to $\nu_{0}$ for some small $\epsilon>0$. Part (a) continues to apply and it follows that

$$
v_{\epsilon}(x)=v(x)+\frac{\epsilon}{x+\epsilon^{2}}
$$

has at most two sign changes on $(0, \infty)$. Since $v_{\epsilon}(0)>0$ for sufficiently small $\epsilon>0$, the set where $v_{\epsilon}<0$ is then at most a single interval. Letting $\epsilon \rightarrow 0+$, we then arrive at a contradiction in case $v$ is negative on $\left(0, x_{1}\right) \cup\left(x_{2}, \infty\right)$.

This proves part (b) of the lemma in all cases. 


\subsubsection{Second step: Monotonicity of $v$ on $\operatorname{supp}\left(\nu^{+}\right)$}

In order to make the induction step in the iterated balayage argument that follows we need the following behavior of $v$ on the parts where it is positive.

Lemma 3.3. Under the same conditions as in Lemma 3.2 where we let $x_{1}, x_{2}$ be as in part (b) of Lemma 3.2, the following hold.

(a) $v^{\prime}$ and $(x v)^{\prime}$ have at most two zeros in $(0, \infty)$.

(b) If $0<x_{1}<x_{2}$ then $x \mapsto v(x)$ is strictly decreasing for $x \in\left(0, x_{1}\right)$.

(c) If $x_{1}<x_{2}<\infty$ then $x \mapsto x v(x)$ is strictly increasing for $x \in\left(x_{2}, \infty\right)$.

Proof. (a) The proof is similar to the proof of part (a) of Lemma 3.2. Note that from 3.4

$$
v^{\prime}(x)=-\int_{0}^{\infty} \frac{d \rho(s)}{(x+s)^{2}}+\frac{A_{1}}{\left(x+q^{-1}\right)^{2}} .
$$

We let $s_{1}=q^{-1}$ and write $v^{\prime}=f_{0}^{\prime}-f_{1}^{\prime}+f_{2}^{\prime}$ with $f_{0}, f_{1}, f_{2}$ as in (3.5) and then

$$
\operatorname{det}\left[f_{j}^{\prime}\left(x_{k}\right)\right]_{j, k=0}^{2}=-A_{1} \int_{0}^{s_{1}} d \rho\left(s_{0}\right) \int_{s_{1}}^{\infty} d \rho\left(s_{2}\right) \operatorname{det}\left[\frac{1}{\left(x_{k}+s_{j}\right)^{2}}\right]_{j, k=0}^{2} .
$$

A Maple calculation shows that

$$
\begin{array}{r}
\operatorname{det}\left[\frac{1}{\left(x_{k}+s_{j}\right)^{2}}\right]_{j, k=0}^{2}=\frac{\prod_{0 \leq j<k \leq 2}\left(x_{k}-x_{j}\right) \prod_{0 \leq j<k \leq 2}\left(s_{j}-s_{i}\right)}{\prod_{j=0}^{2} \prod_{k=0}^{2}\left(x_{k}+s_{j}\right)^{2}} \\
\quad \times P\left(x_{0}, x_{1}, x_{2} ; s_{0}, s_{1}, s_{2}\right)
\end{array}
$$

where $P$ is a homogeneous degree six polynomial in the six variables whose coefficients (with respect to the monomial basis) are all positive. Thus $P>0$ when all its arguments are $>0$, and it follows that (3.10) is negative, and in particular non-zero, whenever $0<x_{0}<x_{1}<x_{2}$. Thus $v^{\prime}$ cannot have more than two positive zeros.

The proof for $(x v)^{\prime}$ is similar, since

$$
(x v)^{\prime}=\int_{0}^{\infty} \frac{s d \rho(s)}{(x+s)^{2}}-\frac{A_{1}}{q\left(x+q^{-1}\right)^{2}}
$$


which has a similar form as (3.9), and the same argument applies.

(b) Suppose $0<x_{1}<x_{2}$. Then $v\left(x_{1}\right)=0$ and $v(x) \geq 0$ for $x \in\left(0, x_{1}\right)$ by Lemma 3.2 (b). Also $v(x) \leq 0$ for $x \in\left(x_{1}, x_{2}\right)$.

Since $v(x) \rightarrow 0$ as $x \rightarrow \infty$, there is a global negative minimum, say at $x^{*}>x_{1}$, where the derivative vanishes and changes sign. According to part (a), there is at most one other sign change of the derivative. If this were in the interval $\left(0, x_{1}\right)$ then it would correspond to a local maximum of $v$ on the interval $\left(0, x_{1}\right)$. Then we modify $v$ to $v_{\epsilon}$ as in $(3.8)$ in the proof of Lemma 3.2. Part (a) applies to $v_{\epsilon}$ and it follows that $v_{\epsilon}^{\prime}$ has at most two sign changes. For $\epsilon>0$ small enough one sign change is close to $x^{*}$, say at $x^{*}(\epsilon)$, and $v_{\epsilon}$ has its global minimum there. Since $v_{\epsilon}^{\prime}(0)<0$ for $\epsilon>0$ sufficiently small, there can be no sign change of $v_{\epsilon}^{\prime}$ in $\left(0, x^{*}(\epsilon)\right)$, and letting $\epsilon \rightarrow 0+$ we find that $v^{\prime}$ has no sign change in $\left(0, x^{*}\right)$. Thus $v$ is strictly decreasing in $\left(0, x_{1}\right)$ as claimed in part $(\mathrm{b})$.

(c) The proof for part (c) is similar. Suppose $x_{1}<x_{2}<\infty$, so that $v\left(x_{2}\right)=0$ and $v(x) \geq 0$ for $x \in\left(x_{2}, \infty\right)$ by Lemma 3.2 (b).

Since $x v(x) \rightarrow 0$ as $x \rightarrow 0$, there is a global minimum of $x \mapsto x v(x)$, at $x^{*} \in\left(x_{1}, x_{2}\right)$ say, where the derivative is zero and changes sign. There is at most one more zero by part (a). If there were a sign change of $(x v)^{\prime}$ in $\left(x_{2}, \infty\right)$, then that would give us a maximum of $x \mapsto x v(x)$ on $\left(x_{2}, \infty\right)$. We again modify $v$ to $v_{\epsilon}$ as in (3.8). Part (a), applied to $v_{\epsilon}$, tells us that $\left(x v_{\epsilon}\right)^{\prime}$ has at most two sign changes on $(0, \infty)$. For small $\epsilon>0$ one sign change is close to $x^{*}$, say at $x^{*}(\epsilon)$ where $x v_{\epsilon}(x)$ has its global minimum. Since

$$
\lim _{x \rightarrow \infty}\left(x v_{\epsilon}(x)\right)^{\prime}>0
$$

for small enough $\epsilon>0$, this derivative then has no sign change in $\left(x^{*}(\epsilon), \infty\right)$ and therefore $x v_{\epsilon}(x)$ is strictly increasing in $\left(x^{*}(\epsilon), \infty\right)$. Letting $\epsilon \rightarrow 0+$ it follows that $x v(x)$ increases strictly in $\left(x^{*}, \infty\right)$, and a fortiori in $\left(x_{2}, \infty\right)$.

\subsubsection{Third step: Iterated balayage}

In the final step we use the iterated balayage to complete the proof of Proposition 3.1. We take $\nu_{1}=\nu$ where $\nu$ is the signed measure on $[0, \infty)$ with density (3.2). If $\nu_{1} \geq 0$ then $\nu_{1}=\mu$ and we are in the full interval support (FIS) case $\Sigma=[0, \infty)$.

In the rest of the proof we assume that $\nu_{1}$ is not a positive measure. Then iteratively we construct the sequence $\left(\nu_{k}\right)_{k}$ as in 3.3 . Inductively we then have $\int d \nu_{k}=\int d \nu_{1}=\frac{1}{2} \int \sigma$ and $\mu \leq \nu_{k}^{+}$for every $k$, and in particular

$$
\Sigma \subset \operatorname{supp}\left(\nu_{k}^{+}\right) \text {. }
$$


The sequence $\left(\int d \nu_{k}^{+}\right)_{k}$ decreases and if $\int d \nu_{k}^{-}$tends to 0 as $k \rightarrow \infty$, then $\nu_{k}^{+} \rightarrow \mu$ in the sense of weak ${ }^{*}$ convergence of measures on $[0, \infty]$. In the present situation (with the help of Lemmas 3.2 and 3.3 we can prove that this is indeed the case.

Lemma 3.4. For every $k$ we have

(a) $\operatorname{supp}\left(\nu_{k}^{+}\right)=\overline{\left(0, x_{1, k}\right)} \cup \overline{\left(x_{2, k}, \infty\right)}$ for some $0 \leq x_{1, k}<x_{2, k} \leq \infty$, while $\operatorname{supp}\left(\nu_{k}^{-}\right) \subset\left[x_{1, k}, x_{2, k}\right]$, and

(b) $\nu_{k}$ has a density $\frac{v_{k}(x)}{2 \pi \sqrt{x}}$ where $x \mapsto v_{k}(x)$ strictly decreases on $\left(0, x_{1, k}\right)$ and $x \mapsto x v_{k}(x)$ strictly increases on $\left(x_{2, k}, \infty\right)$.

Assuming that Lemma 3.4 holds, we complete the proof of Proposition 3.1 as follows. The measures $\left(\nu_{k}^{+}\right)$converges to $\mu$, and $\operatorname{supp}(\mu)=$ $\overline{\left(0, x_{1}\right)} \cup \overline{\left(x_{2}, \infty\right)}$ where $x_{1}=\lim _{k} x_{1, k}$ and $x_{2}=\lim _{k} x_{2, k}$. This establishes Proposition 3.1 .

Proof of Lemma 3.4. For $k=1$, the statements (a) and (b) are contained in Lemmas 3.2 and 3.3 .

Suppose the lemma holds for a certain $k \geq 1$. Let us assume that $0<x_{1, k}<x_{2, k}<\infty$. We use the fact that the balayage of a delta mass $\delta_{t}$ at $t \in\left(x_{1, k}, x_{2, k}\right)$ onto $\left[0, x_{1, k}\right] \cup\left[x_{2, k}, \infty\right)$ has the density

$$
\frac{c(t)}{2 \pi \sqrt{x}} \frac{x+b(t)}{|x-t|} \frac{1}{\sqrt{\left(x-x_{1, k}\right)\left(x-x_{2, k}\right)}}
$$

with positive constants $b(t)>0$ and $c(t)>0$. Then $\operatorname{Bal}\left(\nu_{k}^{-}, \operatorname{supp}\left(\nu_{k}^{+}\right)\right)$has the density

$$
\frac{1}{\pi \sqrt{x}} \frac{1}{\sqrt{\left(x-x_{1, k}\right)\left(x-x_{2, k}\right)}} \int c(t) \frac{x+b(t)}{|x-t|} d \nu_{1}^{-}(t), \quad x \in\left(0, x_{1, k}\right) \cup\left(x_{2, k}, \infty\right) .
$$

In view of 3.3 and the induction hypothesis we then obtain that $\nu_{k+1}$ has the density $\frac{v_{k+1}(x)}{2 \pi \sqrt{x}}$ with

$$
v_{k+1}(x)=v_{k}(x)-\frac{1}{\sqrt{\left(x-x_{1, k}\right)\left(x-x_{2, k}\right)}} \int_{x_{1, k}}^{x_{2, k}} c(t) \frac{x+b(t)}{|x-t|} d \nu_{k}^{-}(t),
$$

for $x \in\left(0, x_{1, k}\right) \cup\left(x_{2, k}, \infty\right)$. Also by the induction hypothesis $v_{k}(x)$ is strictly decreasing on $\left(0, x_{1, k}\right)$. The other term in the right-hand side of (3.11) (including the minus-sign) is also decreasing on $\left(0, x_{1, k}\right)$, since each 
of the factors $\frac{1}{\sqrt{x_{1, k}-x}}, \frac{1}{\sqrt{x_{2, k}-x}}, x+b(t)$, and $\frac{1}{t-x}$ is positive and strictly increasing for $x \in\left(0, x_{1, k}\right)$ as $0<x_{1, k}<t<x_{2, k}$ and $b(t)>0$. Also note that $c(t) d \nu_{k}^{-}(t)$ is a positive measure on $\left[x_{1, k}, x_{2, k}\right]$.

Thus $v_{k+1}$ is strictly decreasing on $\left(0, x_{1, k}\right)$. Then it is either fully negative on $\left(0, x_{1, k}\right)$, in which case we take $x_{1, k+1}=0$, or $v_{k+1}$ is positive on some interval $\left(0, x_{1, k+1}\right)$ with $0<x_{1, k+1}<x_{1, k}$ and $v_{k+1}$ is negative on $\left(x_{1, k+1}, x_{1, k}\right)$.

Similar arguments show that $x v_{k+1}(x)$ is strictly increasing on $\left(x_{2, k}, \infty\right)$. Here we need to observe that each of the factors $\frac{\sqrt{x}}{\sqrt{x-x_{1, k}}}, \frac{\sqrt{x}}{\sqrt{x_{2, k}-x}}$, and $\frac{x+b(t)}{t-x}$ decreases on $\left(x_{2, k}, \infty\right)$. Thus $v_{k+1}$ is either fully negative there, in which case we put $x_{2, k+1}=\infty$, or $v_{k+1}$ is positive on some interval $\left(x_{2, k+1}, \infty\right)$ with $x_{2, k}<x_{2, k+1}<\infty$ and $v_{k+1}$ is negative on $\left(x_{2, k}, x_{2, k+1}\right)$.

Parts (a) and (b) of the lemma are thus proved for $k+1$ in case $0<$ $x_{1, k}<x_{2, k}<\infty$. If $x_{1, k}=0$ or $x_{2, k}=\infty$, then there is an analogous reasoning (which is simpler). The lemma follows by induction.

\subsection{Proof of part (b)}

\subsubsection{Definitions}

We first define maps $M, \widetilde{M}$ and $M_{j}$ between signed measures and vectors of signed measures that will be used in the proof of part (b) of Theorem 2.3 .

Definition 3.5. (a) For a signed measure $\sigma$ on $(-\infty, 0]$ with $0<\int d \sigma<$ $\infty$ we define $M(\sigma)=\mu$ as the measure on $[0, \infty)$ that minimizes

$$
I(\mu)-I(\mu, \sigma)
$$

among $\mu \geq 0$ with $\int d \mu=\frac{1}{2} \int d \sigma$.

(b) Similarly, for a signed measure $\sigma$ on $[0, \infty)$ with $0<\int d \sigma<\infty$ we define $\widetilde{M}(\sigma)=\mu$ as the measure on $(0,-\infty]$ that minimizes 3.12 among $\mu \geq 0$ with $\int d \mu=\frac{1}{2} d \sigma$.

(c) Consider vectors $\vec{\nu}=\left(\nu_{0}, \ldots, \nu_{r+1}\right)$ of signed measures of length $r+2$, such that $\nu_{j}$ is supported on $(-1)^{j}[0, \infty)$ for $j=0,1, \ldots, r+1$ and $0<\int d \nu_{j-1}+\int d \nu_{j+1}<\infty$ for $j=1, \ldots, r$. For such $\vec{\nu}$ we define

$$
M_{j} \vec{\nu}=\left(\nu_{0}, \ldots, \nu_{j-1}, \widehat{\nu}_{j}, \nu_{j+1}, \ldots, \nu_{r+1}\right), \quad j=1, \ldots, r,
$$

where

$$
\widehat{\nu}_{j}= \begin{cases}M\left(\nu_{j-1}+\nu_{j+1}\right), & \text { if } j \text { is odd } \\ \widetilde{M}\left(\nu_{j-1}+\nu_{j+1}\right), & \text { if } j \text { is even }\end{cases}
$$


with $M$ and $\widetilde{M}$ as defined in parts (a) and (b).

Some remarks are in order.

Remark 3.6. (a) The measures $M(\sigma)$ and $\widetilde{M}(\sigma)$ in parts (a) and (b) of Definition 3.5 are minimizers of weakly admissible equilibrium problems. The minimizers uniquely exist [27].

(b) If $\sigma \geq 0$ then $\mu=M(\sigma)$ is the balayage measure $\mu=\frac{1}{2} \operatorname{Bal}(\sigma,[0, \infty))$. In this case we have a monotonicity result

$$
0 \leq \sigma \leq \widetilde{\sigma} \Longrightarrow M(\sigma) \leq M(\widetilde{\sigma})
$$

for measures $\sigma$ and $\widetilde{\sigma}$ on $(-\infty, 0]$.

(c) Similarly

$$
0 \leq \sigma \leq \widetilde{\sigma} \Longrightarrow \widehat{M}(\sigma) \leq \widehat{M}(\widetilde{\sigma})
$$

for measures $\sigma$ and $\widetilde{\sigma}$ on $[0, \infty)$.

(d) The maps are positive homogeneous in the sense that $M(c \sigma)=c M(\sigma)$, $\widehat{M}(c \sigma)=c \widehat{M}(\sigma)$ and $M_{j}(c \vec{\nu})=c M_{j}(\vec{\nu})$ if $c>0$.

(e) If $\left(\mu_{1, t}, \ldots, \mu_{r, t}\right)$ is the solution of the VEP of Definition 2.1 for some $q>0$ and $t \in(0,1)$, and $\vec{\mu}_{t}=\left(\mu_{0, t}, \mu_{1, t}, \ldots, \mu_{r, t}, \mu_{r+1, t}\right)$, then

$$
M_{j}\left(\vec{\mu}_{t}\right)=\vec{\mu}_{t}, \quad j=1, \ldots, r .
$$

That is, $\vec{\mu}_{t}$ is a common fixed point for the mappings $M_{j}$. It is the only common fixed point among vectors $\vec{\mu}$ with $\mu_{0}$ and $\mu_{r+1}$ given by (2.4.

\subsubsection{Monotonicity of $M$}

We are going to apply $M$ only to positive measures and to signed measures whose negative part is a single point mass at $-q^{-1}$ (as in Proposition 3.1). We need the extension of the monotonicity result (3.14) to such signed measure. It could be that the monotonicity result is valid more generally, but we do not consider it here since this is all we need for our present purposes.

For such signed measures $\sigma$ we have the information about the supports of $M(\sigma)$ from Proposition 3.1 , and we also rely on the iterated balayage that was used in the proof of Proposition 3.1 . 
Lemma 3.7. Let $\sigma \leq \widetilde{\sigma}$ be signed measure on $(-\infty, 0]$ with $0<\int d \sigma<$ $\int d \widetilde{\sigma}<\infty$ whose negative parts are single point masses at $-q^{-1}$ only. Then $M(\sigma) \leq M(\widetilde{\sigma})$.

Proof. Under the assumptions of the lemma, the signed measures take the form $\sigma=-A \delta_{-q^{-1}}+\sigma^{+}$and $\widetilde{\sigma}=-\widetilde{A} \delta_{-q^{-1}}+\widetilde{\sigma}^{+}$with $A \geq \widetilde{A} \geq 0$, and $0 \leq \sigma^{+} \leq \widetilde{\sigma}^{+}$. We write $\mu=M(\sigma)$ and $\widetilde{\mu}=M(\widetilde{\sigma})$.

We recall the iterated balayage algorithm from the proof of Proposition 3.1, see in particular Lemma 3.4, and we apply it to the signed measure $\widetilde{\sigma}$. That is, we start with $\nu_{1}=\frac{1}{2} \operatorname{Bal}(\widetilde{\sigma},[0, \infty))$, and from there we construct the sequence $\left(\nu_{k}\right)_{k}$ inductively by

$$
\nu_{k+1}=\nu_{k}^{+}-\operatorname{Bal}\left(\nu_{k}^{-}, \operatorname{supp}\left(\nu_{k}^{+}\right)\right), \quad k=1,2, \ldots
$$

Then $\left(\nu_{k}\right)$ converges to $\widetilde{\mu}=M(\widetilde{\sigma})$ as was shown in the proof of Lemma 3.4 .

Next we define a second sequence $\left(\rho_{k}\right)_{k}$ by $\rho_{1}=\frac{1}{2} \operatorname{Bal}(\sigma,[0, \infty))$, and

$$
\rho_{k+1}=\rho_{k}^{+}-\operatorname{Bal}\left(\rho_{k}^{-}, \operatorname{supp}\left(\nu_{k}^{+}\right)\right), \quad k=1,2, \ldots
$$

Since $\sigma \leq \widetilde{\sigma}$ we have $\rho_{1} \leq \nu_{1}$, and then by induction it easily follows that $\rho_{k} \leq \nu_{k}$ for every $k$. Note that we deviate from the earlier construction by taking in (3.17) the balayage of $\rho_{k}^{+}$onto $\operatorname{supp}\left(\nu_{k}^{+}\right)$and not onto $\operatorname{supp}\left(\rho_{k}^{+}\right)$. Since $\operatorname{supp}\left(\nu_{k}^{+}\right) \supset \operatorname{supp}\left(\rho_{k}^{+}\right)$, we however still find (by induction) that $\mu=$ $M(\sigma) \leq \rho_{k}^{+}$for every $k$. Then $\rho_{\infty}=\lim _{k \rightarrow \infty} \rho_{k}$ is a signed measure with $\rho_{\infty} \leq \widetilde{\mu}$ and $\mu \leq \rho_{\infty}^{+}$. Thus $\mu \leq \widetilde{\mu}$ as claimed in the lemma.

\subsection{3 $M$-convexity}

We need two more definitions. Note that $M$-convexity is not a standard terminology, but it is introduced here to help the exposition.

Definition 3.8. Let $\vec{\nu}$ be as in Definition 3.5 (c), and let $M_{j}$ be as in (3.13). Then we say that $\vec{\nu}$ is $M$-convex if

$$
\vec{\nu} \leq M_{j}(\vec{\nu}) \quad \text { for every } j=1, \ldots, r .
$$

Definition 3.9. The set $\mathcal{M}_{q}$ contains those vectors $\vec{\nu}=\left(\nu_{0}, \nu_{1}, \ldots, \nu_{r+1}\right)$ satisfying

- $\nu_{j}$ is a positive measure on $(-1)^{j-1}[0, \infty)$ for $j=1, \ldots, r+1$,

- $\nu_{0}=-A \delta_{-q^{-1}}$ for some $A<\int d \nu_{2}$. 
Then we have the following properties.

Lemma 3.10. Suppose $\vec{\nu} \in \mathcal{M}_{q}$.

(a) Then $M_{j}(\vec{\nu}) \in \mathcal{M}_{q}$ for every $j=1, \ldots, r$.

(b) If $\vec{\nu} \leq \vec{\rho} \in \mathcal{M}_{q}$ then $M_{j}(\vec{\nu}) \leq M_{j}(\vec{\rho})$ for every $j=1, \ldots, r$.

(c) If $\vec{\nu}$ is $M$-convex then so is $M_{j} \vec{\nu}$ for every $j=1, \ldots, r$.

(d) If $\vec{\nu}$ is $M$-convex and $c>0$ then $\vec{\nu}+\left(c \delta_{-q^{-1}}, 0,0, \ldots, 0\right)$ is $M$-convex.

(e) If $\vec{\nu}$ is $M$-convex and $\rho \geq 0$ is a measure on $(-1)^{r}[0, \infty)$ then $\vec{\nu}+$ $(0,0, \ldots, 0, \rho)$ is $M$-convex.

Proof. (a) Obvious.

(b) This follows from the monotonicity of $M$ and $\widetilde{M}$ on positive measures, see (3.14) and (3.15), and the monotonicity of $M$ on signed measures whose negative part only contains a point mass at $-q^{-1}$, see Lemma 3.7 .

(c) Since $\vec{\nu}$ is $M$-convex we have $\vec{\nu} \leq M_{k} \vec{\nu}$ for every $k$. The maps $M_{k}$ and $M_{j}$ commute if $|j-k| \neq 1$. Thus it follows from part (b) that

$$
M_{j} \vec{\nu} \leq M_{j} M_{k} \vec{\nu}=M_{k} M_{j} \vec{\nu}, \quad k \neq\{j-1, j+1\} .
$$

For $k \in\{j-1, j+1\}$ we can verify by direct inspection that the inequality between $M_{j} \vec{\nu}$ and $M_{k} M_{j} \vec{\nu}$ also holds. The two vectors only differ at positions $k=j \pm 1$, which for $M_{j} \vec{\nu}$ is equal to $\nu_{j \pm 1}$, and for $M_{j \pm 1} M_{j} \vec{\nu}$ it is $M\left(\nu_{j \pm 2}+\widehat{\nu}_{j}\right)$ or $\widetilde{M}\left(\nu_{j \pm 2}+\widehat{\nu}_{j}\right)$ (depending on the parity of $j$ ) with $\widehat{\nu}_{j}$ as in 3.13 ). By $M$ convexity of $\vec{\nu}$ we have $\nu_{j} \leq \widehat{\nu}_{j}$ and by the monotonicity properties of $M$ and $\widetilde{M}$ and $M$-convexity once more, we have

$$
\nu_{j-1} \leq M\left(\nu_{j \pm 2}+\nu_{j}\right) \leq M\left(\nu_{j \pm 2}+\widehat{\nu}_{j}\right) \quad \text { if } j \text { is odd }
$$

with $M$ replaced by $\widetilde{M}$ if $j$ is even. This proves $M_{j} \vec{\nu} \leq M_{k} M_{j} \vec{\nu}$ also in case $|j-k|=1$ and part (c) follows.

(d) and (e) are straightfoward verifications.

\subsubsection{Proof of Theorem 2.3 (b)}

Proof. Let us take $0<s<t<1$. Then we have to show that $s \mu_{j, s} \leq t \mu_{j, t}$ for every $j=0,1, \ldots, r+1$. This is clear for $j=0$ and $j=r+1$ due to the definitions (2.4). 
Write $\vec{\mu}_{s}=\left(\mu_{0, s}, \mu_{1, s}, \ldots, \mu_{r+1, s}\right)$ and similarly for $\vec{\mu}_{t}$. Then by the definition of the operators $M_{j}$, we have

$$
M_{j}\left(s \vec{\mu}_{s}\right)=s \vec{\mu}_{s}, \quad M_{j}\left(t \vec{\mu}_{t}\right)=t \vec{\mu}_{t} .
$$

see also Remark 3.6 (d) and (e).

Now we put

$$
\begin{aligned}
\vec{\nu}_{1} & =s \vec{\mu}_{s}+\left((t-s) \delta_{-q^{-1}}, 0, \ldots, 0,(t-s) \delta_{(-1)^{r} q}\right) \\
& =\left(t \mu_{0, t}, s \mu_{1, s}, s \mu_{2, s}, \ldots, s \mu_{r, s}, t \mu_{r+1, t}\right) .
\end{aligned}
$$

This is the vector $s \vec{\mu}_{s}$ with the 0 th and $r+1$ st components replaced by those of $t \vec{\mu}_{t}$. Then $s \vec{\mu}_{s} \leq \vec{\nu}_{1}$ and $\vec{\nu}_{1}$ is $M$-convex by Lemma 3.10 (d) and (e) and the fact that $s \vec{\mu}_{s}$ is $M$-convex.

We choose an infinite sequence $\left(j_{k}\right)_{k}$ in $\{1, \ldots, r\}$ where we make sure that every $j$ in $\{1, \ldots, r\}$ appears an infinite number of times in the sequence. Then we define a sequence $\left(\vec{\nu}_{k}\right)_{k}$ by

$$
\vec{\nu}_{k+1}=M_{j_{k}}\left(\vec{\nu}_{k}\right) \quad \text { for } k=1,2, \ldots
$$

Inductively we find that each $\vec{\nu}_{k}$ is $M$-convex by Lemma 3.10(c) and the fact that $\vec{\nu}_{1}$ is $M$-convex. Then $\vec{\nu}_{k} \leq \vec{\nu}_{k+1}$ for every $k$.

Also by induction it is easy to show that

$$
\int d\left(\vec{\nu}_{k}\right)_{j} \leq t+j-1
$$

for every $k$ and for every $j$. Thus the sequence $\left(\vec{\nu}_{k}\right)$ is increasing with a componentwise limit $\vec{\nu}_{k} \rightarrow \vec{\nu}_{\infty}$, as $k \rightarrow \infty$ (with convergence in weak*sense).

If $j=j_{k}$ is even, then

$$
\left(\vec{\nu}_{k+1}\right)_{j}=M\left(\left(\vec{\nu}_{k}\right)_{j-1}+\left(\vec{\nu}_{k}\right)_{j+1}\right) .
$$

while for $j$ is odd we have to replace $M$ by $\widetilde{M}$.

If we take the limit $k \rightarrow \infty$ along the subsequence for which $j_{k}=j$, then it follows from this that $M_{j}\left(\vec{\nu}_{\infty}\right)=\vec{\nu}_{\infty}$ for every $j$. Since the 0th and $r+1$ st components are those of $t \vec{\mu}_{t}$, we we conclude that $\vec{\nu}_{\infty}=t \vec{\mu}_{t}$, see also Remark 3.6 (e).

We combine the inequalities to find

$$
s \vec{\mu}_{s} \leq \vec{\nu}_{1} \leq \cdots \leq \vec{\nu}_{k} \leq \cdots \leq \vec{\nu}_{\infty}=t \vec{\mu}_{t}
$$

which indeed shows that $s \mu_{j, s} \leq t \mu_{j, t}$ for every $j$. 


\subsection{Proof of part (c)}

\subsubsection{An equivalent equilibrium problem}

For $\theta>0$ and a measure $\mu$ on $[0, \infty)$ we write

$$
I_{\theta}(\mu)=\iint \log \frac{1}{\left|x^{\theta}-y^{\theta}\right|} d \mu(x) d \mu(y)
$$

which we may call the $\theta$-energy of $\mu$. For $\theta=1$ it reduces to the usual logarithmic energy $I(\mu)$ of $\mu$.

Proposition 3.11. Let $\left(\mu_{1}, \ldots, \mu_{r}\right)$ be the solution of the VEP of Definition 2.1 with parameters $q>0$ and $0<t<1$. Then the first component $\mu_{1}$ minimizes

$$
\frac{1}{2} I(\nu)+\frac{1}{2} I_{\theta}(\nu)+\int V(x) d \nu(x)
$$

with $\theta=\frac{1}{r}$ and

$$
V(x)=-\frac{1-t}{t} \log \left(x+q^{-1}\right)+\frac{r+t}{t} \log \left(x^{1 / r}+q^{1 / r}\right)
$$

among all probability measures $\nu$ on $[0, \infty)$.

Energy functionals of the form 3.20 appeared before in the context of Muttalib-Borodin ensembles [6, 41]. These are joint probability densities for $n$ particles on the positive real line of the form

$$
\frac{1}{Z_{n}} \prod_{1 \leq i<j \leq n}\left(x_{i}-x_{j}\right)\left(x_{i}^{\theta}-x_{j}^{\theta}\right) \prod_{j=1}^{n} e^{-n V\left(x_{j}\right)}, \quad \text { all } x_{j}>0
$$

where $\theta>0$ is a positive constant and $Z_{n}$ is a normalization factor. In the large $n$ limit the particles are distributed according to the minimizer of (3.20), see [10, 19] and see [9, 20, 32, 40] for some recent contributions on Muttalib-Borodin ensembles (3.22). We use the characterization of $\mu_{1}$ via the equilibrium problem from Proposition 3.11 in the proofs of parts (c) and (d) of Theorem 2.3 .

The proof of Proposition 3.11 follows along the lines of the proof of Theorem 1.1 in 29 .

Proof of Proposition 3.11. For a probability measure $\nu$ on $[0, \infty)$ we define

$$
J(\nu)=\min _{\nu_{2}, \ldots, \nu_{r}}\left(\sum_{j=2}^{r} I\left(\nu_{j}\right)-\sum_{j=1}^{r} I\left(\nu_{j}, \nu_{j+1}\right)\right)
$$


where $\nu_{1}=\nu$ and $\nu_{r+1}=\mu_{r+1}=\frac{r+t}{t} \delta_{(-1)^{r} q}$. The minimization is over all $\nu_{2}, \ldots, \nu_{r}$ satisfying the support condition 2.2 and the total mass condition (2.3), i.e., $\operatorname{supp}\left(\nu_{j}\right) \subset \Delta_{j}$ and $\nu_{j}\left(\Delta_{j}\right)=1+\frac{j-1}{t}$ for $j=2, \ldots, r$.

This is again a weakly admissible vector equilibrium problem, similar to the VEP from Definition 2.1, and it has a unique solution. It is simpler to solve, since only positive measures are involved and we can be sure that the minimizers $\nu_{2}, \ldots, \nu_{r}$ have full supports, with the property

$$
2 U^{\nu_{j}}=U^{\nu_{j-1}}+U^{\nu_{j+1}} \quad \text { on } \Delta_{j}, \quad \text { for } j=2, \ldots, r,
$$

see also (2.12).

From (3.24) we obtain

$$
\begin{aligned}
I\left(\nu_{j}\right) & =\int U^{\nu_{j}} d \nu_{j}=\frac{1}{2} \int\left(U^{\nu_{j-1}}-U^{\nu_{j+1}}\right) d \nu_{j} \\
& =\frac{1}{2} I\left(\nu_{j-1}, \nu_{j}\right)+\frac{1}{2} I\left(\nu_{j}, \nu_{j+1}\right), \quad j=2, \ldots, r .
\end{aligned}
$$

Hence from 3.23

$$
\begin{aligned}
J(\nu) & =-\frac{1}{2} I\left(\nu, \nu_{2}\right)-\frac{1}{2} I\left(\nu_{r}, \nu_{r+1}\right) \\
& =-\frac{1}{2} \int U^{\nu_{2}} d \nu-\frac{r+t}{2 t} U^{\nu_{r}}\left((-1)^{r} q\right),
\end{aligned}
$$

where $\nu_{2}$ and $\nu_{r}$ are from the minimizer $\left(\nu_{2}, \ldots, \nu_{r}\right)$ associated with $\nu$. We are going to calculate $U^{\nu_{2}}$ and $U^{\nu_{r}}$.

We first do this for a point mass $\nu=\delta_{p}$ with $p>0$, and the general case is obtained by averaging over $p$. So let $\left(\nu_{2}, \ldots, \nu_{r}\right)$ be the minimizer associated with $\nu=\nu_{1}=\delta_{p}$. We use the Riemann surface $\mathcal{S}$ with $r$ sheets $\mathcal{S}^{(j)}, j=1, \ldots, r$, given by

$$
\begin{array}{ll}
\mathcal{S}^{(1)} & =\mathbb{C} \backslash(-\infty, 0], \\
\mathcal{S}^{(j)} & =\mathbb{C} \backslash \mathbb{R}, \\
\mathcal{S}^{(r)} & =\mathbb{C} \backslash\left((-1)^{r}[0, \infty)\right) .
\end{array}
$$

Sheet $S^{(j)}$ is connected to sheet $S^{(j-1)}$ along the cut $\Delta_{j}=(-1)^{j}[0, \infty)$ for $j=2, \ldots, r$. We add a point at infinity to obtain a compact Riemann surface. 

follows.

We define a function $\Psi$ on $\mathcal{S}$ by its restriction $\Psi^{(j)}$ to the $j$ th sheet as

$$
\begin{aligned}
& \Psi^{(1)}(z)=\frac{z}{z-p}-z \int \frac{d \nu_{2}(s)}{z-s} \\
& \Psi^{(j)}(z)=z \int \frac{d \nu_{j}(s)}{z-s}-z \int \frac{d \nu_{j+1}(s)}{z-s}, \quad \text { for } j=2, \ldots, r-1, \\
& \Psi^{(r)}(z)=z \int \frac{d \nu_{r}(s)}{z-s}-\frac{r+t}{t} \frac{z}{z-(-1)^{r} q} .
\end{aligned}
$$

The conditions (3.24) imply that $\Psi$ is meromorphic on $\mathcal{S}$ with poles at $z=p$ on the first sheet and at $z=(-1)^{r} q$ on the $r$ th sheet, see also the discussion after Definition 2.6 that shows why $\Phi$ is meromorphic on $\mathcal{R}$. [The construction of $\Psi$ is similar to that of $\Phi$.] Due to the total masses of the measures we have $\Psi(z) \rightarrow-\frac{1}{t}$ as $z \rightarrow \infty$.

The Riemann surface 3.26 has a simple parametrization $z=w^{r}$, and in the $w$ variable the poles are at $w=p^{1 / r}$ and $w=-q^{1 / r}$. Taking into account the residues at the poles and the behavior at infinity, we find that

$$
\Psi(z)=\frac{1}{r} \frac{p^{1 / r}}{w-p^{1 / r}}+\frac{r+t}{r t} \frac{q^{1 / r}}{w+q^{1 / r}}-\frac{1}{t}, \quad z=w^{r} .
$$

Observe also that $\Psi(z)=0$ for $z=w=0$.

Specifying (3.28) to the first sheet, and recalling (3.27) we find

$$
\begin{aligned}
\int \frac{d \nu_{2}(s)}{z-s} & =\frac{1}{z-p}-\frac{1}{z} \Psi^{(1)}(z) \\
& =\frac{1}{z-p}-\frac{1}{r z} \frac{p^{1 / r}}{z^{1 / r}-p^{1 / r}}-\frac{r+t}{r t z} \frac{q^{1 / r}}{z^{1 / r}+q^{1 / r}}-\frac{1}{t z}
\end{aligned}
$$

with principal branch of the fractional powers. We integrate with respect to $z$ and find after straightforward calculation

$$
\int \log (z-s) d \nu_{2}(s)=\log (z-p)-\log \left(z^{1 / r}-p^{1 / r}\right)+\frac{r+t}{t} \log \left(z^{1 / r}+q^{1 / r}\right)
$$

There is no constant of integration since both sides behave as $\left(1+t^{-1}\right) \log z+$ $o(1)$ as $z \rightarrow \infty$. The real part of (3.30) gives us the logarithmic potential

$$
U^{\nu_{2}}(z)=\log \left|\frac{z^{1 / r}-p^{1 / r}}{z-p}\right|-\frac{r+t}{t} \log \left|z^{1 / r}+q^{1 / r}\right| .
$$


An analogous calculation, based on (3.28) and the expression (3.27) of $\Psi$ on the $r$ th sheet, leads to the logarithmic potential of $\nu_{r}$,

$$
U^{\nu_{r}}(z)=\frac{r+t}{r} \log \left|\frac{z^{1 / r}+q^{1 / r}}{z-(-1)^{r} q}\right|-\log \left|z^{1 / r}-p^{1 / r}\right|
$$

with the branch of the $r$ th root that is analytic on $\mathbb{C} \backslash \Delta_{r}$ and that is real and negative for real $z \in \mathbb{C} \backslash \Delta_{r}$. However, we emphasize that $p^{1 / r}$ and $q^{1 / r}$ always denote the positive $r$ th roots. Similarly when we write $x^{1 / r}$ with $x>0$ as for example in (3.20) and in (3.34) below.

Thus (3.31) and (3.32) give the logarithmic potentials of $\nu_{2}$ and $\nu_{r}$ associated with $\delta_{p}$. Associated with a general probability measure $\nu=\nu_{1}$ on $[0, \infty)$, we then have measures $\nu_{2}$ and $\nu_{r}$ whose logarithmic potentials are obtained from averaging (3.31) and (3.32) over $p$, that is

$$
U^{\nu_{2}}(z)=\int \log \left|\frac{z^{1 / r}-x^{1 / r}}{z-x}\right| d \nu(x)-\frac{r+t}{t} \log \left|z^{1 / r}+q^{1 / r}\right|
$$

and

$$
U^{\nu_{r}}(z)=\frac{r+t}{r} \log \left|\frac{z^{1 / r}+q^{1 / r}}{z-(-1)^{r} q}\right|-\int \log \left|z^{1 / r}-x^{1 / r}\right| d \nu(x) .
$$

From 3.33 we obtain

$$
I\left(\nu, \nu_{2}\right)=I(\nu)-I_{1 / r}(\nu)-\frac{r+t}{t} \int \log \left|x^{1 / r}+q^{1 / r}\right| d \nu(x),
$$

and from (3.34) we obtain, noting that $z^{1 / r}$ in (3.34) is negative for $z \in$ $\mathbb{R} \backslash \Delta_{r}$, and thus in particular for $z=(-1)^{r} q$,

$$
\begin{aligned}
U^{\nu_{r}}\left((-1)^{r} q\right) & =\frac{r+t}{t} \log \lim _{x \rightarrow q}\left|\frac{x^{1 / r}-q^{1 / r}}{x-q}\right|-\int \log \left|-q^{1 / r}-x^{1 / r}\right| d \nu(x) \\
& =\frac{r+t}{t} \log \left(\frac{1}{r} q^{1 / r-1}\right)-\int \log \left(x^{1 / r}+q^{1 / r}\right) d \nu(x) .
\end{aligned}
$$

Using (3.35) and 3.36$)$ in 3.25 we obtain

$$
\begin{aligned}
J(\nu)=-\frac{1}{2} I(\nu)+\frac{1}{2} I_{1 / r}(\nu)+\frac{r+t}{t} \int & \log \left(x^{1 / r}+q^{1 / r}\right) d \nu(x) \\
& -\frac{1}{2}\left(\frac{r+t}{t}\right)^{2} \log \left(\frac{1}{r} q^{1 / r-1}\right) .
\end{aligned}
$$


Finally, comparing (3.23) with the energy functional 2.5 we obtain for a given $\nu$ on $[0, \infty)$ that

$$
\min _{\nu_{2}, \ldots, \nu_{r}} \mathcal{E}\left(\nu, \nu_{2}, \ldots, \nu_{r}\right)=I(\nu)-I\left(\nu, \mu_{0}\right)+J(\nu)
$$

so that in view of (3.37) and noting that $\mu_{0}$ is given by (2.4)

$$
\begin{aligned}
\min _{\nu_{2}, \ldots, \nu_{r}} \mathcal{E}\left(\nu, \nu_{2}, \ldots, \nu_{r}\right) & =\frac{1}{2} I(\nu)+\frac{1}{2} I_{1 / r}(\nu) \\
-\frac{1-t}{t} \int \log \left(x+q^{-1}\right) d \nu(x) & +\frac{r+t}{t} \int \log \left(x^{1 / r}+q^{1 / r}\right) d \nu(x) \\
& -\frac{1}{2}\left(\frac{r+t}{t}\right)^{2} \log \left(\frac{1}{r} q^{1 / r-1}\right) .
\end{aligned}
$$

The left-hand side of (3.38) as a functional on probability measures $\nu$ on $[0, \infty)$ attains its minimum at $\nu=\mu_{1}$. Since the last term on the right in (3.38) is only a constant, independent of $\nu$, the proposition follows.

\subsubsection{Minimum of $V$ is in the support}

In the next step we discuss a general fact about the minimizer for a MuttalibBorodin type energy functional (3.20) with $\theta>0$, and where $V:[0, \infty) \rightarrow \mathbb{R}$ is continuous with

$$
\liminf _{x \rightarrow \infty}(V(x)-(1+\theta) \log x) \geq-\infty .
$$

Under this condition there is a unique probability measure $\mu$ on $[0, \infty)$ that minimizes 3.20.

The following is well-known for the case $\theta=1$, but apparently has not been observed for general $\theta$.

Lemma 3.12. If $x_{0} \geq 0$ is such that $V\left(x_{0}\right)=\min _{x \geq 0} V(x)$ then $x_{0} \in \operatorname{supp}(\mu)$ where $\mu$ is the probability measure that minimizes (3.20).

Proof. In this proof we use the notation

$$
U_{\theta}^{\mu}(x)=\int \log \frac{1}{\left|x^{\theta}-s^{\theta}\right|} d \mu(s) .
$$

The minimizer $\mu$ satisfies, for some constant $\ell$,

$$
U^{\mu}(x)+U_{\theta}^{\mu}(x)+V(x) \begin{cases}=\ell, & \text { on } \operatorname{supp}(\mu), \\ \geq \ell & \text { on }[0, \infty) .\end{cases}
$$


Now,

$$
h(x)=U^{\mu}(x)+U_{\theta}^{\mu}(x)=\int \log \frac{1}{|x-s|} d \mu(s)+\int \log \frac{1}{\left|x^{\theta}-s^{\theta}\right|} d \mu(s),
$$

extends into the complex plane where we use the principal branch of $x^{\theta}$, i.e., with a branch cut along $(-\infty, 0]$. Then $h$ is harmonic in $\mathbb{C} \backslash((-\infty, 0] \cup$ $\operatorname{supp}(\mu))$ and it tends to $-\infty$ as $|x| \rightarrow \infty$. By the maximum principle for harmonic functions, the maximum of $h$ is attained on $\operatorname{supp}(\mu) \cup(-\infty, 0]$ only.

For $x>0$ and $s>0$, it is easy to see that $|-x-s|>|x-s|$ and $\left|(-x)^{\theta}-s^{\theta}\right|=\left|x^{\theta} e^{\pi i \theta}-s^{\theta}\right|>\left|x^{\theta}-s^{\theta}\right|$. Therefore $U^{\mu}(-x)<U^{\mu}(x)$ and $U_{\theta}^{\mu}(-x)<U_{\theta}^{\mu}(x)$ for $x>0$ which means that $h(-x)<h(x)$ for $x>0$, and therefore the maximum of $h$ is not attained on $(-\infty, 0]$.

Thus the maximum of $h$ is attained on $\operatorname{supp}(\mu)$, say at $x_{1} \in \operatorname{supp}(\mu)$.

If $x_{0} \notin \operatorname{supp}(\mu)$, then $h\left(x_{0}\right)<h\left(x_{1}\right)$, and since $V\left(x_{0}\right) \leq V\left(x_{1}\right)$ by the assumption in the lemma, we have a strict inequality

$$
h\left(x_{0}\right)+V\left(x_{0}\right)<h\left(x_{1}\right)+V\left(x_{1}\right) .
$$

Since $x_{1} \in \operatorname{supp}(\mu)$ the right-hand side of 3.40 is equal to $\ell$, by the equality in (3.39) It follows that

$$
U^{\mu}\left(x_{0}\right)+U_{\theta}^{\mu}\left(x_{0}\right)+V\left(x_{0}\right)<\ell
$$

which contradicts the inequality in 3.39$)$. Therefore $x_{0} \in \operatorname{supp}(\mu)$.

\subsubsection{Proof of part (c) of Theorem 2.3.}

In view of Proposition 3.11 and Lemma 3.12 it is enough to show that the external field (3.21) attains its minimum at $x=0$ in case $0<q<1$. This is what we do in the next lemma, and then part (c) follows.

Lemma 3.13. Let $V$ be given by (3.21) with $0<q<1$. Then for every $t \in(0,1)$ it is true that

$$
V(0)=\min _{x \geq 0} V(x) .
$$

Proof. Note that $\left(x^{1 / r}+q^{1 / r}\right)^{r} \geq x+q$ for $x \geq 0$ by the binomial theorem, so that by (3.21)

$$
\begin{aligned}
& t V(x)-t V(0) \\
& \quad \geq-(1-t) \log \left(x+q^{-1}\right)+\frac{r+t}{r} \log (x+q)-\left(2-t+\frac{t}{r}\right) \log q .
\end{aligned}
$$


Since $0<q<1$, we have $x+q \geq q^{2} x+q$ for $x \geq 0$ and therefore we can estimate 3.41 further to obtain

$$
\begin{aligned}
& t V(x)-t V(0) \\
& \quad \geq-(1-t) \log \left(x+q^{-1}\right)+\frac{r+t}{r} \log \left(q^{2} x+q\right)-\left(2-t+\frac{t}{r}\right) \log q .
\end{aligned}
$$

This means $V(x)-V(0) \geq\left(1+\frac{1}{r}\right) \log (q x+1)$ and the lemma follows.

\subsection{Proof of part (d)}

The measure $\mu_{1}$ minimizes $I(\mu)-I\left(\mu, \mu_{0}+\mu_{2}\right)$ among all probability measures on $[0, \infty)$. Thus it is the equilibrium measure in the external field $-U^{\mu_{0}+\mu_{2}}$ which is real analytic on $[0, \infty)$. Then it follows from [14 that $\mu_{1}$ is absolutely continuous with respect to Lebesgue measure with a density that is real analytic in the interior of its support.

From [14] it also follows that the density at the endpoint $x_{1}$ (in BIS and TIS cases) behaves as $\approx c\left(x_{1}-x\right)^{\frac{1}{2}+2 N}$ as $x \rightarrow x_{1}-$, for a certain non-negative integer $N$. From the iterated balayage it can be seen that $N=0$. Indeed, the algorithm in section 3.1.4 gives us the sequence of signed measures $\left(\nu_{k}\right)_{k}$ with densities that are such that $\sqrt{x} \frac{d \nu_{k}}{d x}$ is positive and strictly decreasing on $\left[0, x_{1}\right]$ by Lemma 3.4 (b). The proof of that lemma (see (3.11)) actually shows that the decrease gets stronger as $k$ increases. Since $\nu_{k} \rightarrow \mu_{1}$ as $k \rightarrow \infty$, it then follows that the density of $\mu_{1}$ cannot have a zero derivative at $x_{1}$, and thus it vanishes as a square root at $x_{1}$ in BIS and TIS cases.

Similarly, the density vanishes as a square root at $x_{2}$ in UIS and TIS cases.

\section{Proof of Theorem 2.8}

\subsection{Properties of $\Phi$}

We start by listing a number of properties of the meromorphic function $\Phi$ from Definition 2.6.

\subsubsection{Zeros and poles}

Lemma 4.1. Let $q>0$. The function $z \Phi$ is a degree 2 meromorphic function on the Riemann surface $\mathcal{R}$ with the following properties. 
(a) $z \Phi \rightarrow 1$ as $z \rightarrow \infty$ on any of the sheets.

(b) It has simple poles at $z=-q^{-1}$ on the first sheet and at $z=(-1)^{r} q$ on the last sheet, and no other poles.

(c) Suppose one of the BIS, TIS, or FIS cases, so that $z \in \operatorname{supp}\left(\mu_{1}\right)$. Then $z \Phi$ has simple zeros at $z=0$ and at a point $x_{0}$ on the first sheet with

$$
-q^{-1}<x_{0}<0
$$

and no other zeros. In UIS case, there are two points on the Riemann surface with $z=0$ and $z \Phi$ has a simple zero at each of these.

Proof. We already noted that $\Phi$ is meromorphic on $\mathcal{R}$, see the discussion after Definition 2.6. Thus also $z \Phi$ is meromorphic on $\mathcal{R}$. In part (b) we show that it has two simple poles, and no other poles, and therefore its degree is two.

(a) From 2.19 we have $z F_{j}(z) \rightarrow \mu_{j}\left(\Delta_{j}\right)$ as $z \rightarrow \infty$. In view of the total massses (2.3) of the measures and the definition (2.21), part (a) follows.

(b) From 2.20 and 2.21 we see that

$$
\begin{aligned}
\Phi^{(1)}(z) & =t F_{1}(z)+\frac{1-t}{z+q^{-1}} \\
& =t \int \frac{d \mu_{1}(s)}{z-s}+\frac{1-t}{z+q^{-1}}, \\
\Phi^{(r+1)}(z) & =-t F_{r}(z)+\frac{r+t}{z-(-1)^{r} q},
\end{aligned}
$$

and so $z \Phi$ has simple poles at $-q^{-1}$ on the first sheet and at $(-1)^{r} q$ on the $r+1$-st sheet.

There is no pole at $z=\infty$ because of part (a). There is no pole at $z=0$ either, since the form (2.19) of $F_{j}$ as a Stieltjes transform, easily implies that $z F_{j}(z) \rightarrow 0$ as $z \rightarrow 0$. Thus also $z \Phi \rightarrow 0$ as $z \rightarrow 0$. There are no other candidates for poles, and therefore the degree is two.

(c) We already remarked in part (b) that $z \Phi$ vanishes when $z=0$. In UIS case there are two points on the Riemann surface with $z=0$. In that case both of these are simple zeros, and there are no other zeros, since the degree is two.

In BIS, TIS, and FIS cases, there is only one point $z=0$, and it is at most a double zero of $z \Phi$. Then $z^{\frac{1}{r+1}}$ is the local coordinate, and $z$ (as a 
function on the Riemann surface) has a zero of order $r+1$ at $z=0$. Hence $\Phi$ has a pole at $z=0$ of order $\geq r-1$, and so $\Phi$ is unbounded at $z=0$ (we may assume $r \geq 2$ ). Looking on the first sheet, we conclude from (4.2) that $F_{1}$ is unbounded at $z=0$, and it dominates the behavior of $\Phi^{(1)}$ as $z \rightarrow 0$. Since $F_{1}(z)<0$ for negative real $z$, it then follows that $\Phi^{(1)}(z)$ is negative for negative $z$ close to 0 .

From (4.2) we also see that $\Phi^{(1)}(z) \rightarrow+\infty$ as $z \rightarrow-q^{-1}+$, as the residue at the pole is positive. Thus $\Phi^{(1)}$ changes sign on the interval $\left(-q^{-1}, 0\right)$ and hence there is a zero, say at $x_{0} \in\left(-q^{-1}, 0\right)$. Then $x_{0}$ is also a zero of $z \Phi$, and we conclude that both $z=x_{0}$ and $z=0$ are simple zeros, and these are the only zeros, as the degree of $z \Phi$ is two.

\subsubsection{Proof of Theorem 2.3 (e)}

Proof. Suppose we are in one of the BIS, TIS, or FIS cases, so that $0 \in$ $\operatorname{supp}\left(\mu_{1}\right)$. Then $z=0$ is a simple zero of $z \Phi$, by part (c) of Lemma 4.1. Since $z^{\frac{1}{r+1}}$ is a local coordinate, we find that $\Phi$ has a pole of order $r$ at $z=0$. From 4.2 we then get for some non-zero constant $C$,

$$
F_{1}(z)=C z^{-\frac{r}{r+1}}\left(1+O\left(z^{\frac{1}{r+1}}\right)\right) \quad \text { as } z \rightarrow 0
$$

and the fractional powers have their branch cut along $[0, \infty)$. By the Stieltjes inversion formula

$$
\frac{d \mu_{1}}{d x}=-\frac{1}{\pi} \lim _{\delta \rightarrow 0+} \operatorname{Im} F_{1}(x+i \delta), \quad x>0,
$$

and $(2.13)$ follows.

Finally, 2.14 follows from 2.13) and the symmetry between $q$ and $1 / q$, see Remark 2.4.

Remark 4.2. The behavior (2.13) is characteristic for the density of minimizers of Muttalib-Borodin type energy functionals as in (3.20). This was proved by Claeys and Romano [10, Remark 1.9] under general conditions on the external field, which however do not cover the case (3.21).

\subsubsection{Critical points}

We need to know about the critical points, by which we mean the ramification points of $z \Phi$. There is no ramification at $z=0$ or $z=\infty$, and so we may alternatively characterize the critical points as those points where the derivative of $z \Phi^{(j)}(z)$ vanishes for some $j=1, \ldots, r+1$. 


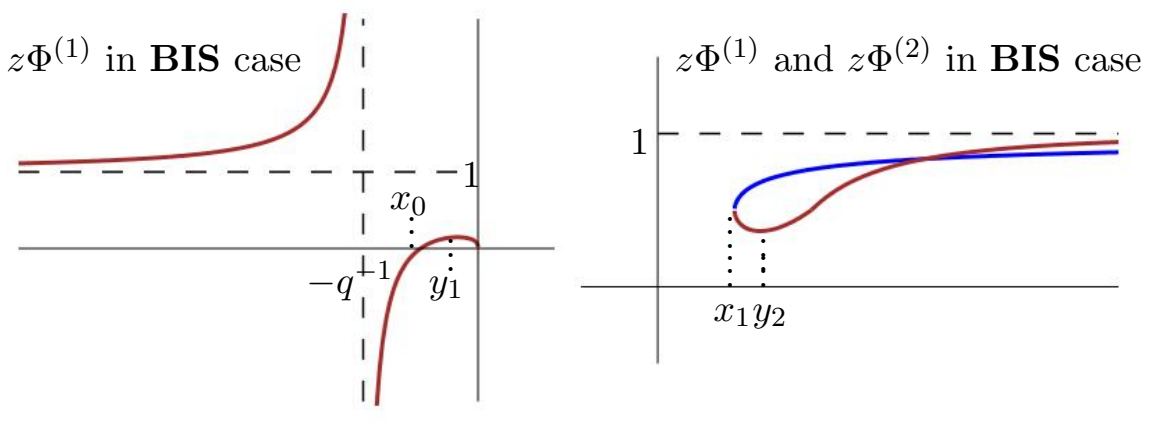

Figure 5: Sketches of the graph of $z \Phi$ in BIS case. The left panel shows the graph of $z \Phi^{(1)}(z)$ on the negative real line where it has a pole at $-q^{-1}$, a zero at $x_{0}$ and a local maximum at $y_{1}$. The right panel shows the graphs of $z \Phi^{(1)}(z)$ (in brown) and $z \Phi^{(2)}$ (in blue) on the interval $\left[x_{1}, \infty\right)$. The graph of $z \Phi^{(1)}(z)$ has a local minimum at $y_{2}$, while the graph of $z \Phi^{(2)}(z)$ is strictly increasing. Both graphs tend to 1 at infinity. The graph of $z \Phi^{(r+1)}(z)$ is as in the right panel of Figure 7 below.

Since $z \Phi$ has degree 2 the Riemann-Hurwitz formula [46] tells us that there are two critical points in the BIS and FIS (genus zero) cases, and four critical points in the TIS (genus one) case. The following lemma says that they are all real and on the first sheet.

We fix $0<q<1$ and we continue to use $x_{1}, x_{2}$ as in Theorem 2.3 (a) depending on the various cases, and $x_{0}$ for the zero of $\Phi$ on the first sheet as in Lemma 4.1 (c).

Lemma 4.3. Let $0<q<1$. The critical points of $z \Phi$ are on the real part of the first sheet of the Riemann surface.

(a) In all cases there is a critical point $y_{1}$ with $y_{1} \in\left(x_{0}, 0\right)$.

(b) In $\boldsymbol{B I S}$ case there is one more critical point $y_{2} \in\left(x_{1}, \infty\right)$.

(c) In $\boldsymbol{T I S}$ case there are three more critical points. A critical point $y_{0} \in$ $\left(-\infty,-q^{-1}\right)$ and two critical points $y_{2}, y_{3} \in\left(x_{1}, x_{2}\right)$ with $y_{2}<y_{3}$.

(d) In $\boldsymbol{F I S}$ case there is one more critical point $y_{0} \in\left(-\infty,-q^{-1}\right)$. 

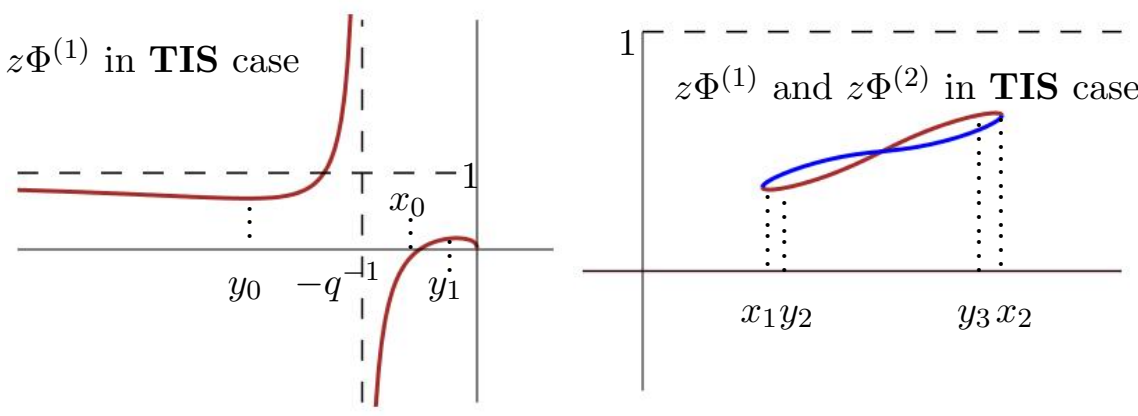

Figure 6: Sketches of the graph of $z \Phi$ in TIS case. The left panel shows the graph of $z \Phi^{(1)}(z)$ on the negative real line where it has a pole at $-q^{-1}$, zeros at $x_{0}$ and 0 , a local minimum at $y_{0}$, and a local maximum at $y_{1}$. The right panel shows the graphs of $z \Phi^{(1)}(z)$ (in brown) and $z \Phi^{(2)}$ (in blue) on the interval $\left[x_{1}, x_{2}\right]$. The graph of $z \Phi^{(1)}(z)$ has a local minimum at $y_{2}$ and a local maximum at $y_{3}$, while the graph of $z \Phi^{(2)}(z)$ is strictly increasing. The graph of $z \Phi^{(r+1)}(z)$ is as in the right panel of Figure 7 below.

Proof. (a) By Lemma 4.1 (c) $z \Phi^{(1)}(z)$ has zeros at $z=x_{0}$ and at $z=0$, and in between it is real and positive. So there is a local maximum, and the point $y_{1} \in\left(x_{0}, 0\right)$ where it is attained is a critical point in all cases.

The proofs of parts (b)-(d) rely on an inspection of the graph of $z \Phi$ on the real part of the Riemann surface (that is, on the part where both $z$ and $\Phi$ are real), see Figures 5, 6, and 7 for sketches of the graphs in the various cases. We infer the following about $z \Phi$ from the behavior at the poles and at infinity,

- every value in $(-\infty, 0)$ is attained once in $\left(-q^{-1}, x_{0}\right)$ on the first sheet and once between 0 and $(-1)^{r} q$ on the last sheet,

- every value in $(1, \infty)$ is attained once in $\left(-\infty,-q^{-1}\right)$ on the first sheet and once between $(-1)^{r} q$ and infinity on the last sheet.

Since $z \Phi$ has degree two, the values in $(-\infty, 0)$ and $(1, \infty)$ are attained nowhere else on the Riemann surface. In particular

$$
\begin{aligned}
& 0<z \Phi^{(1)}(z)<1, \text { and } \\
& 0<z \Phi^{(2)}(z)<1,
\end{aligned} \quad\left\{\begin{array}{l}
\text { for } z \in\left[x_{1}, \infty\right) \\
\text { for } z \in\left[x_{1}, x_{2}\right] \text { in BIS case, }
\end{array}\right.
$$




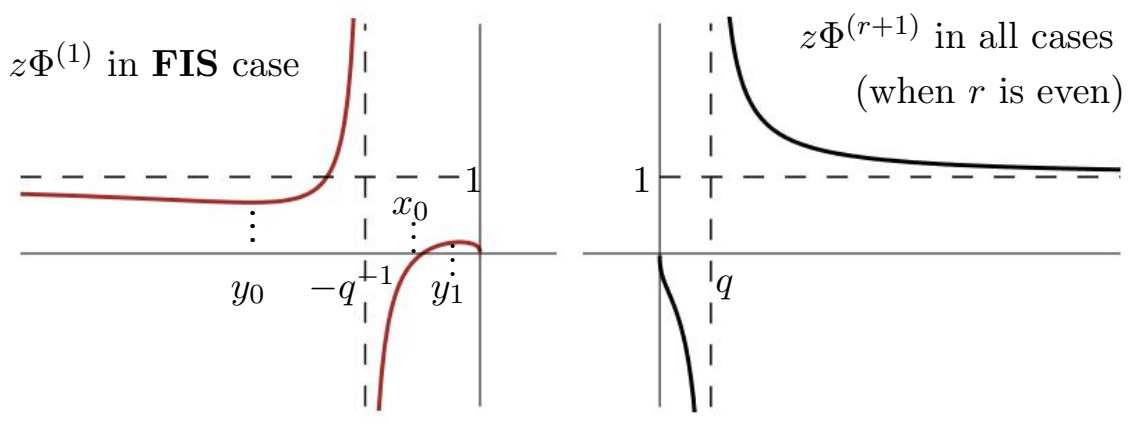

Figure 7: Sketches of the graph of $z \Phi$ in FIS case. The left panel shows the graph of $z \Phi^{(1)}(z)$ on $(-\infty, 0]$ where it has a pole at $-q^{-1}$, zeros at $x_{0}$ and 0 , a local minimum at $y_{0}$, and a local maximum at $y_{1}$. The right panel shows the graph of $z \Phi^{(r+1)}(z)$ on $(-1)^{r}[0, \infty)$ which has a pole at $(-1)^{r} q$. The figure is for $r=2$ and it has the same features for all cases.

see the right panels of Figures 5 and 6 .

From the fact that the density of $\mu_{1}$ vanishes as a square root at the endpoints $x_{1}, x_{2}$ see Theorem 2.3 (d), it follows that

$$
F_{1}^{\prime}(z)=-\int \frac{1}{(z-x)^{2}} d \mu_{1}(x) \rightarrow-\infty
$$

as $z \rightarrow x_{1}+$ or $z \rightarrow x_{2}-$. Hence by 2.21) we also have

$$
\left(z \Phi^{(1)}(z)\right)^{\prime} \rightarrow-\infty \quad \text { and } \quad\left(z \Phi^{(2)}(z)\right)^{\prime} \rightarrow+\infty
$$

as $z \rightarrow x_{1}+$ (in BIS and TIS cases) or $z \rightarrow x_{2}-$ (in TIS case).

(b) In BIS case we noted in 4.4 that $z \Phi^{(1)}(z)$ takes the value $x_{1} \Phi^{(1)}\left(x_{1}\right) \in$ $(0,1)$ at $x_{1}$, and by (4.6) it starts to decrease if $z \in\left(x_{1}, \infty\right)$ increases. Since it tends to the value 1 at infinity, there will be a local minimum, say at $y_{2} \in\left(x_{1}, \infty\right)$. This is a critical point, and part (b) follows.

It also follows that $z \Phi^{(2)}(z)$ strictly increases for $z \in\left[x_{1}, \infty\right)$ since in BIS case there are no further critical points.

(c) In TIS case we first observe that

$$
\frac{d}{d z}\left(z F_{1}(z)\right)=-\int \frac{x}{(z-x)^{2}} d \mu_{1}(x)<0, \quad z \in\left(x_{1}, x_{2}\right)
$$




$$
\frac{d}{d z}\left(z F_{2}(z)\right)=-\int \frac{x}{(z-x)^{2}} d \mu_{2}(x)>0, \quad z \in\left(x_{1}, x_{2}\right) .
$$

The difference in sign is due to the fact that $\mu_{1}$ is supported on $[0, \infty)$, while $\mu_{2}$ is supported on $(-\infty, 0]$. Thus by 2.21

$$
\left(z \Phi^{(2)}(z)\right)^{\prime}>0 \quad \text { for } z \in\left(x_{1}, x_{2}\right),
$$

and therefore $z \Phi^{(2)}(z)$ strictly increases on $\left(x_{1}, x_{2}\right)$ and there are no critical points in $\left(x_{1}, x_{2}\right)$ on the second sheet. We also conclude

$$
x_{1} \Phi^{(1)}\left(x_{1}\right)<x_{2} \Phi^{(1)}\left(x_{2}\right)
$$

but $z \Phi^{(1)}(z)$ will not be monotonic on $\left[x_{1}, x_{2}\right]$ due to 4.6$)$. Instead it will start to decrease at $x_{1}$ to a local minimum, say at $y_{2}$, and then increases to a local maximum, say at $y_{3}$, and then again decreases. This gives us the critical points $y_{2}<y_{3}$ in $\left(x_{1}, x_{2}\right)$. We already know $y_{1} \in\left(-x_{0}, 0\right)$.

The final critical point is in $\left(-\infty,-q^{-1}\right)$, and this follows from the observation that

$$
z F_{1}(z)=z \int \frac{d \mu_{1}(x)}{z-x}=1+\int \frac{x d \mu_{1}(x)}{z-x}
$$

with $\int \frac{x d \mu_{1}(x)}{z-x}<0$ for $z \in(-\infty, 0]$ and

$$
\int \frac{x d \mu_{1}(x)}{z-x}=-C z^{-\frac{1}{r+1}}\left(1+O\left(z^{-\frac{1}{r+1}}\right) \text { as } z \rightarrow-\infty\right.
$$

with a positive constant $C>0$. Also $z F_{0}(z)=\left(1-t^{-1}\right)+O\left(z^{-1}\right)$ as $z \rightarrow \infty$, so that by 2.21 .

$$
z \Phi^{(1)}(z)=1+\int \frac{x d \mu_{1}(x)}{z-x}+O\left(z^{-1}\right)
$$

as $z \rightarrow-\infty$, where the second term is negative for $z<0$ and it dominates the $O\left(z^{-1}\right)$ term as $z \rightarrow-\infty$. Therefore $z \Phi^{(1)}(z)$ decreases on an interval $\left(-\infty, y_{0}\right)$ for some $y_{0} \in\left(-\infty,-q^{-1}\right)$, it reaches a local minimum at $y_{0}$ and then increases to $+\infty$ as $z \rightarrow-q^{-1}-$. See also Figure 6 .

(d) In FIS case the expansions 4.9 and 4.10 remain valid, as does the conclusion that $z \Phi^{(1)}(z)$ has a local minimum at some $y_{0} \in\left(-\infty,-q^{-1}\right)$, and $y_{0}$ is a critical point.

After these preparations we turn to the proof of Theorem 2.8 . 


\subsection{Proof of part (a)}

Proof. For $x \in \operatorname{supp}\left(\mu_{1}\right)$, we have by 2.21 and the Stieltjes inversion formula

$$
x \operatorname{Im} \Phi_{ \pm}^{(1)}(x)=x t \operatorname{Im}\left(F_{1}\right)_{ \pm}(x)=\mp \frac{x t}{\pi} \frac{d \mu_{1}(x)}{d x} .
$$

Here the subscript \pm denotes the limiting value from the upper $(+)$ or lower $(-)$ half plane. Then by 2.23 we find $\operatorname{supp}\left(\mu_{1}\right) \subset U$.

from the Cauchy-Riemann equations and the definition (2.23) of $U$, we obtain that the parts of the real line where $z \Phi^{(1)}(z)$ is real and decreasing belong to $U$, while those parts where $z \Phi^{(1)}(z)$ is real and increasing do not belong to $U$. Then in view of the behavior of $z \Phi^{(1)}(z)$ on the realline that we see in Figures 5, 6, 7, and the fact that $\operatorname{supp}\left(\mu_{1}\right) \subset U$, we conclude that

$$
U \cap(\mathbb{R} \cup\{\infty\})= \begin{cases}{\left[y_{1}, y_{2}\right]} & \text { in BIS case } \\ {\left[-\infty, y_{0}\right] \cup\left[y_{1}, y_{2}\right] \cup\left[y_{3}, \infty\right]} & \text { in TIS case } \\ {\left[-\infty, y_{0}\right] \cup\left[y_{1}, \infty\right]} & \text { in FIS case }\end{cases}
$$

In particular $-q^{-1} \notin U$. This proves part (a).

\subsection{Proof of part (b)}

Proof. Since the $y_{j}$ 's are critical points, we have that $z \Phi^{(1)}(z)$ is also real on certain contours that emanate from each $y_{j}$ into the complex plane. These contours are going to be the boundary $\partial U$ of $U$.

The labelling of the critical points in Lemma 4.3 is such that $y_{j}$ is a local minimum of $z \Phi^{(1)}(z)$ if $j$ is even, and a local maximum if $j$ is odd, when we restrict to the real line. It means that $z \Phi^{(1)}(z)$ is real and increasing on $\partial U$ when we move away from $y_{j}$ with $j$ odd, and decreasing from $y_{j}$ with $j$ even.

Noting that

$$
y_{1} \Phi^{(1)}\left(y_{1}\right)<y_{2} \Phi^{(1)}\left(y_{2}\right) \text { in BIS case, }
$$

we conclude that the part of $\partial U$ that emanates from $y_{1}$ will end at $y_{2}$ in BIS case. Since $\Sigma_{1}=\operatorname{supp}\left(\mu_{1}\right) \subset U$, we also see that $\partial U$ consists of a simple closed contour surrounding $\Sigma_{1}$ and $U$ is a bounded simply connected domain in the BIS case. 


\subsection{Proof of part (c)}

Proof. In TIS case, we have four critical points and instead of 4.13 we have

$$
y_{1} \Phi^{(1)}\left(y_{1}\right)<y_{2} \Phi^{(1)}\left(y_{2}\right)<y_{3} \Phi^{(1)}\left(y_{3}\right)<y_{0} \Phi^{(1)}\left(y_{0}\right) \quad \text { in TIS case. }
$$

Then $\partial U$ consists of two closed contours, one containing $y_{1}$ and $y_{2}$, and one containing $y_{0}$ and $y_{3}$. Both closed contours go around $\Sigma_{1}$. It follows that $U$ has two components, namely the bounded domain that is enclosed by the inner component of $\partial U$, and the unbounded domain that is outside of the outer component of $\partial U$. This proves part (c).

\subsection{Proof of part $(d)$}

Proof. In FIS case we have two critical points $y_{0}<y_{1}<0$ with

$$
y_{1} \Phi^{(1)}\left(y_{1}\right)<y_{0} \Phi^{(1)}\left(y_{0}\right), \quad \text { in FIS case. }
$$

Then $\partial U$ is a closed contour containing $y_{0}$ and $y_{1}$, and $U$ is the domain that is exterior to this contour.

\subsection{Proof of part (e)}

Proof. We know from Theorem 2.3 (b) that $t \mapsto t \mu_{j, t}$ increases with $t$ for every $j$, and $t \mu_{j, t}$ has total mass $t+j-1$. Then

$$
\rho_{j}=\rho_{j, t}=\frac{\partial\left(t \mu_{j, t}\right)}{\partial t}, \quad j=0, \ldots, r+1
$$

is a probability measure on $\operatorname{supp}\left(\mu_{j}\right)$ for every $j$. In particular $\rho_{0}=\delta_{-q^{-1}}$ by 2.3 . .

Thus by differentiating (2.21) for $j=1$ with respect to $t$,

$$
\frac{\partial \Phi^{(1)}(z)}{\partial t}=\int \frac{d \rho_{1}(x)}{z-x}-\frac{1}{z+q^{-1}} .
$$

Since $\rho_{0}$ and $\rho_{1}$ are both probability measures we obtain from this

$$
\frac{\partial\left(z \Phi^{(1)}(z)\right)}{\partial t}=\int \frac{x d \rho_{1}(x)}{z-x}+\frac{q^{-1}}{z+q^{-1}} .
$$


For $\operatorname{Im} z>0$ both terms in the right-hand side of (4.17) have negative imaginary parts (since $x d \rho_{1}(x)$ is a positive measure), while for $\operatorname{Im} z<0$ the two terms have positive imaginary parts. In other words

$$
\frac{\partial}{\partial t} \operatorname{Im}\left(z \Phi^{(1)}(z)\right) \begin{cases}<0 & \text { for } \operatorname{Im} z>0 \\ >0 & \text { for } \operatorname{Im} z<0\end{cases}
$$

Therefore the part in the upper half plane where $\operatorname{Im}\left(z \Phi^{(1)}(z)\right)<0$ increases with $t$. Similarly, the part in the lower half plane where $\operatorname{Im}\left(z \Phi^{(1)}(z)\right)>0$ increass with $t$, which proves part (e) in view of the definition (2.23) of $U$.

\subsection{Proof of part (f)}

Proof. It is clear from $(2.23)$ that $z \Phi^{(1)}(z)$ is real-valued for $z \in \partial U$. The point of part (f) is that $\partial U$ is characterized by (2.24).

Being a meromorphic function on a compact Riemann surface, $\Phi$ satisfies the algebraic equation

$$
\prod_{j=1}^{r+1}\left(\Phi-\Phi^{(j)}(z)\right)=\Phi^{r+1}+\sum_{k=1}^{r+1}(-1)^{k} e_{k}(z) \Phi^{r+1-k}=0
$$

where $e_{k}(z)$ is the $k$ th elementary symmetric function in $\Phi^{(1)}, \ldots, \Phi^{(r+1)}$, i.e.,

$$
e_{k}(z)=\sum_{1 \leq j_{1}<\cdots<j_{k} \leq r+1} \prod_{l=1}^{k} \Phi^{\left(j_{l}\right)}(z) .
$$

Each $e_{k}$ is a rational function of $z \in \mathbb{C}$ with real coefficients and simple poles at $-q^{-1}$ and at $(-1)^{r} q$, due to the simple poles of $\Phi^{(1)}$ and $\Phi^{(r+1)}$ at these respective values, see (4.2) and (4.3). Since $z \Phi^{(j)}(z) \rightarrow 1$ as $z \rightarrow \infty$ for every $j$ by Lemma 4.1 (a), and there are $\left(\begin{array}{c}r+1 \\ k\end{array}\right)$ terms in 4.19 we have

$$
z^{k} e_{k}(z) \rightarrow\left(\begin{array}{c}
r+1 \\
k
\end{array}\right) \quad \text { as } z \rightarrow \infty \text {. }
$$

By Lemma 4.1 (c) $z=0$ is a simple zero of $z \Phi$ on the Riemann surface, and from 4.19) we get that $z^{k} e_{k}(z)$ becomes zero for $z=0$. Thus

$$
z^{k} e_{k}(z)=\left(\begin{array}{c}
r+1 \\
k
\end{array}\right) \frac{z\left(z+A_{k}\right)}{\left(z+q^{-1}\right)\left(z-(-1)^{r} q\right)}
$$


for some real value $A_{k}$.

Using this in (4.18) and clearing denominators by multiplying with $z^{r}(z+$ $\left.q^{-1}\right)\left(z-(-1)^{r} q\right)$ we obtain

$$
\begin{aligned}
& z^{r}\left(z+q^{-1}\right)\left(z-(-1)^{r} q\right) \Phi^{r+1} \\
& +\sum_{k=1}^{r+1}(-1)^{k}\left(\begin{array}{c}
r+1 \\
k
\end{array}\right)\left(z+A_{k}\right)(z \Phi)^{r+1-k}=0 .
\end{aligned}
$$

We separate terms that are polynomial in $z \Phi$ to rewrite 4.20 as (with $\left.A_{0}=q^{-1}-(-1)^{r} q\right)$

$$
\begin{aligned}
& \sum_{k=0}^{r+1}(-1)^{k}\left(\begin{array}{c}
r+1 \\
k
\end{array}\right) A_{k}(z \Phi)^{r+1-k} \\
&=(-1)^{r} z^{-1}(z \Phi)^{r+1}-z \sum_{k=0}^{r+1}(-1)^{k}\left(\begin{array}{c}
r+1 \\
k
\end{array}\right)(z \Phi)^{r+1-k} \\
&=(-1)^{r} z^{-1}(z \Phi)^{r+1}+z(z \Phi-1)^{r+1} .
\end{aligned}
$$

In the last step we used the binomial theorem.

Let $z \in \partial U$. Then $z \Phi^{(1)}(z)$ is real and it satisfies the equation (4.21), which means that the left-hand side is real since each $A_{k}$ is real. Thus the

right-hand side is real as well, and taking imaginary parts we obtain since $z \Phi^{(1)}(z)$ is real,

$$
0=(-1)^{r+1} \frac{\operatorname{Im} z}{|z|^{2}}\left(z \Phi^{(1)}(z)\right)^{r+1}+\operatorname{Im} z\left(z \Phi^{(1)}(z)-1\right)^{r+1}, \quad \text { for } z \in \partial U .
$$

This equation leads to 2.24 whenever $\operatorname{Im} z \neq 0$. Thus 2.24 holds for $z \in \partial U \backslash \mathbb{R}$ and by continuity it also holds for $z \in \partial U \cap \mathbb{R}$.

\section{Proof of Theorem 2.11}

\subsection{Proof of part (a)}

Proof. Since $\mu^{*}$ is the symmetric pullback of the probability measure $\mu_{1}$, it is also a probability measure. That $\mu_{\Omega}$ is a probability measure as well can be seen from the formulas in Lemma 5.2 below, by letting $z \rightarrow \infty$ in either (5.3) (in case $\Omega$ is bounded), or (5.4) (in case $\Omega$ is unbounded). 


\subsection{Proof of part (b)}

Proof. Since $t \mu_{1, t}$ is increasing by Theorem 2.3 (b), also $t \mu_{t}^{*}$ increases with $t$.

The domains $U_{t}$ increase with $t$ by Theorem 2.8 . Then also $\Omega_{t}$ increases with $t$ and then $t \mu_{\Omega, t}$ also increases with $t$, since by $(2.28)$ this is just the spherical area measure $\frac{1}{\pi} \frac{d A(z)}{\left(1+|z|^{2}\right)^{2}}$ restricted to $\Omega_{t}$.

\subsection{Stieltjes transform of $\mu_{\Omega}$}

The proofs of parts (c) and (d) are modelled after the proofs in the paper [11] that deals with the case $r=1$. See in particular the proof of Proposition 4.1 in [11. As a preparation we need the following formula for the spherical Schwarz function from (2.26), which is the analogue of [11, (5.1)].

Lemma 5.1. We have

$$
S(z)=\frac{(1-t) z^{r}}{z^{r+1}+q^{-1}}+t \int \frac{d \mu^{*}(x)}{z-x}
$$

Proof. Since $\mu^{*}$ is the symmetric pullback of $\mu_{1}$ under the mapping $z \mapsto$ $z^{r+1}$, we can easily verify that their logarithmic potentials are related via

$$
U^{\mu^{*}}(z)=\frac{1}{r+1} U^{\mu_{1}}\left(z^{r+1}\right) .
$$

and also, with appropriate branches of the logarithm,

$$
\int \log (z-x) d \mu^{*}(x)=\frac{1}{r+1} \int \log \left(z^{r+1}-x\right) d \mu_{1}(x) .
$$

Taking the $z$-derivative we find

$$
\int \frac{d \mu^{*}(x)}{z-x}=z^{r} F_{1}\left(z^{r+1}\right) .
$$

Then combining (2.26), 4.2), and (5.2), we find (5.1).

The following lemma is the analogue of [11, Lemma 5.2] and its proof is also very similar.

Lemma 5.2. The Stieltjes transform of $\mu_{\Omega}$ satisfies

$$
\begin{aligned}
& \int \frac{d \mu_{\Omega}(x)}{z-x}=\int \frac{d \mu^{*}(x)}{z-x}, \quad z \in \mathbb{C} \backslash \Omega, \\
& \int \frac{d \mu_{\Omega}(x)}{z-x}=-\frac{(1-t) z^{r}}{t\left(z^{r+1}+q^{-1}\right)}+\frac{\bar{z}}{t\left(1+|z|^{2}\right)}, \quad z \in \Omega .
\end{aligned}
$$


Proof. Take $z \in \mathbb{C} \backslash \Omega$ first. Then by $(1.9)$, 2.28), the complex Green's formula, and the property (2.27) of the spherical Schwarz function, we find if $\Omega$ is bounded,

$$
\begin{aligned}
t \int \frac{d \mu_{\Omega}(s)}{z-s} & =\frac{1}{\pi} \int_{\Omega} \frac{d A(s)}{(z-s)\left(1+|s|^{2}\right)^{2}} \\
& =\frac{1}{2 \pi i} \oint_{\partial \Omega} \frac{\bar{s}}{(z-s)\left(1+|s|^{2}\right)} d s \\
& =\frac{1}{2 \pi i} \oint_{\partial \Omega} \frac{S(s)}{z-s} d s \\
& =-\frac{1}{2 \pi i} \oint_{\partial(\mathbb{C} \backslash \Omega)} \frac{S(s)}{z-s} d s .
\end{aligned}
$$

Since the complex Green's formula applies to bounded domains, one has to modify the calculation in case $\Omega$ is unbounded. Then one first makes a cut-off to $\{z \in \Omega|| z \mid \leq R\}$ with a large $R>0$. The Green's formula then produces an additional integral over $|z|=R$, which however tends to zero as $R \rightarrow \infty$, due to the fact that $\frac{S(s)}{z-s}=O\left(s^{-2}\right)$ as $s \rightarrow \infty$. Thus (5.5) also holds in the unbounded case.

The remaining integral in $(5.5)$ is evaluated with the residue theorem for $\mathbb{C} \backslash \Omega$. The spherical Schwarz function $S$ has $r+1$ simple poles at the solutions of $s^{r+1}=-q^{-1}$, the poles are all in $\mathbb{C} \backslash \Omega$, and from (5.1) it can be checked that $S$ has the same residue $\frac{1-t}{r+1}$ at each of the poles. Together they give the contribution

$$
-\frac{1-t}{r+1} \sum_{s^{r+1}=-q^{-1}} \frac{1}{z-s}=-\frac{(1-t) z^{r}}{z^{r+1}+q^{-1}}
$$

to the integral (5.5). There is an additional pole in 5.5 at $s=z$ with the contribution $S(z)$. Finally, note that there is no contribution from infinity in case $\mathbb{C} \backslash \Omega$ is unbounded, since the integrand in $(5.5)$ is $O\left(s^{-2}\right)$ as $s \rightarrow \infty$. In total we get

$$
t \int_{\Omega} \frac{d \mu_{\Omega}(s)}{z-s}=-\frac{(1-t) z^{r}}{z^{r+1}+q^{-1}}+S(z), \quad z \in \mathbb{C} \backslash \Omega,
$$

and (5.4) follows because of 5.1.

Let $z \in \Omega \backslash \partial \Omega$. Take $\varepsilon>0$ such that the disk $D(z, \varepsilon)$ of radius $\varepsilon$ around $z$ is contained in $\Omega$. Then by a calculation similar to (5.5), with complex 
Green's theorem and the spherical Schwarz function

$$
\begin{aligned}
t \int_{\Omega \backslash D(z, \varepsilon)} \frac{d \mu_{\Omega}(s)}{z-s}=- & \frac{1}{2 \pi i} \oint_{\partial(\mathbb{C} \backslash \Omega)} \frac{S(s)}{z-s} d s \\
& -\frac{1}{2 \pi i} \oint_{\partial D(z, \varepsilon)} \frac{\bar{s}}{(z-s)\left(1+|s|^{2}\right)} d s
\end{aligned}
$$

The integral over $\partial(\mathbb{C} \backslash \Omega)$ is again evaluated using the residue theorem, but in the present situation there is no contribution from $s=z$, but only the combined contribution (5.6) from the poles of $S$. The integral over the circle $\partial D(z, \varepsilon)$ (including the prefactor $-\frac{1}{2 \pi i}$ ) tends to $\frac{\bar{z}}{1+|z|^{2}}$ as $\varepsilon \rightarrow 0+$. Thus letting $\varepsilon \rightarrow 0+$ in 5.7 we obtain 5.4 .

\subsection{The log integral of $\mu_{\Omega}$}

We will need the following result in the TIS case for the proof of Lemma 5.5 below.

Lemma 5.3. In $\boldsymbol{F I S}$ and $\boldsymbol{T I S}$ cases we have

$$
U^{\mu_{\Omega}}(0)+\frac{1-t}{(r+1) t} \log q=0 .
$$

In BIS case we have

$$
U^{\mu_{\Omega}}(0)+\frac{1-t}{(r+1) t} \log q=\frac{1}{2 t(r+1)} \int_{x_{1}}^{\infty}\left(\Phi^{(1)}(x)-\Phi^{(2)}(x)\right) d x \leq 0
$$

but we will not prove this as we do not need it for the proof of parts (c) and (d) of Theorem 2.11.

Proof of Lemma 5.3. In the proof we use $\log x=\log |x|+i \arg x$ with $0<$ $\arg x<2 \pi$, and we are going to show that

$$
t \int \log (x) d \mu_{\Omega}(x)=\frac{1-t}{r+1} \log q+t \pi i \quad \text { in FIS and TIS cases }
$$

and then (5.8) will follow by taking the real parts on both sides. The evaluation of 5.9) follows along the lines of the proof of Lemma 5.2 but there is a non-trivial extra step required in the TIS case.

We start with the FIS case. Consider the cut-off domain

$$
\Omega_{R, \delta}=\{z \in \Omega|| z \mid \leq R, \operatorname{dist}(z,[0, \infty))>\delta\}
$$


with large $R>0$ and small $\delta>0$. Due to our definition of the logarithm with the branch cut along $[0, \infty)$ we can apply the complex Green's theorem to the integral over $\Omega_{R, \delta}$ and we find in FIS case

$$
\begin{aligned}
& \frac{1}{\pi} \int_{\Omega_{R, \delta}} \log x \frac{d A(x)}{\left(1+|x|^{2}\right)^{2}}=\frac{1}{2 \pi i} \oint_{\partial \Omega_{R, \delta}} \log s \frac{\bar{s}}{1+|s|^{2}} d s \\
& \rightarrow \frac{1}{2 \pi i} \oint_{\Omega} \log s \frac{\bar{s}}{1+|s|^{2}} d s+\frac{1}{2 \pi i} \oint_{|s|=R} \log s \frac{\bar{s}}{1+|s|^{2}} d s \\
& \quad-\int_{0}^{R} \frac{x}{1+x^{2}} d x
\end{aligned}
$$

as $\delta \rightarrow 0+$. The last term in $(5.10)$ is the combined contribution of the upper and lower sides of the branch cut of the logarithm. It yields

$$
\int_{0}^{R} \frac{x}{1+x^{2}} d x=\frac{1}{2} \log \left(1+R^{2}\right)=\log R+o(1) \quad \text { as } R \rightarrow \infty .
$$

The second integral in the right-hand side of (5.10) is evaluated with parametrization $s=R e^{i \theta}, 0<\theta<2 \pi$, to give

$$
\begin{aligned}
\frac{1}{2 \pi i} \oint_{|s|=R} \log (s) \frac{\bar{s}}{1+|s|^{2}} d s & =\frac{R^{2} \log R}{1+R^{2}}+\frac{R^{2}}{1+R^{2}} \pi i \\
& =\log R+\pi i+o(1) \quad \text { as } R \rightarrow \infty .
\end{aligned}
$$

In the first term we use (2.27) and then evaluate the integral by a residue calculation over $\mathbb{C} \backslash \Omega$. The complement of $\Omega$ consists of $r+1$ disjoint disks in FIS case, see Figure 4 , and $S(s)$ is meromorphic with one simple pole at the solution of $s^{r+1}+q^{-1}$ in each of the disks with residue $\frac{1-t}{r+1}$. Therefore

$$
\begin{aligned}
\frac{1}{2 \pi i} \oint_{\Omega} \log s \frac{\bar{s}}{1+|s|^{2}} d s & =-\frac{1}{2 \pi i} \oint_{\partial(C \backslash \Omega)}(\log s) S(s) d s \\
& =-\frac{1-t}{r+1} \sum_{s: s^{r+1}=-q^{-1}} \log s \\
& =\frac{1-t}{r+1} \log q-(1-t) \pi i
\end{aligned}
$$

Letting $R \rightarrow \infty$ in 5.10 we find from (2.28), 5.11), 5.12 and 5.13 that

$$
\begin{aligned}
t \int \log (x) d \mu_{\Omega}(x) & =\lim _{R \rightarrow \infty} \lim _{\delta \rightarrow 0+} \frac{1}{\pi} \int_{\Omega_{R, \delta}} \log x \frac{d A(x)}{\left(1+|x|^{2}\right)^{2}} \\
& =\frac{1-t}{r+1} \log q+t \pi i
\end{aligned}
$$


as claimed in $(5.9)$ in FIS case.

In TIS case we have to adjust the above calculation in two ways. First, since $\Omega \cap[0, \infty)=\left[0, y_{2}^{*}\right] \cup\left[y_{3}^{*}, \infty\right)$, with $y_{j}^{*}=y_{j}^{1 /(r+1)}$, the integral over $[0, R]$ in 5.10 is replaced by

$$
\left(\int_{0}^{y_{2}^{*}}+\int_{y_{3}^{*}}^{R}\right) \frac{x}{1+x^{2}} d x=-\int_{y_{2}^{*}}^{y_{3}^{*}} \frac{x}{1+x^{2}} d x+\log R+o(1) \quad \text { as } R \rightarrow \infty .
$$

Second, in the evaluation $\sqrt{5.13}$ of the integral over $\Omega$, there is a contribution from the intersection $\left[y_{2}^{*}, y_{3}^{*}\right]$ of $\mathbb{C} \backslash \Omega$ with the positive real line, due to the discontinuity of the logarithm. Instead of (5.13) we get

$$
\frac{1}{2 \pi i} \oint_{\Omega} \log s \frac{\bar{s}}{1+|s|^{2}} d s=\frac{1-t}{r+1} \log q-(1-t) \pi i-\int_{y_{2}^{*}}^{y_{3}^{*}} S(x) d x,
$$

and the result is the formula

$$
t \int \log (x) d \mu_{\Omega}(x)=\frac{1-t}{r+1} \log q+t \pi i-\int_{y_{2}^{*}}^{y_{3}^{*}}\left(S(x)-\frac{x}{1+x^{2}}\right) d x
$$

for the TIS case.

To obtain (5.9) it remains to prove that the integral in the right-hand side vanishes, and we do this by showing the identity (5.14) in Lemma 5.4 below. The right-hand side of $(5.14)$ is zero and to see this we recall (2.21) from which we get

$$
\begin{aligned}
\Phi^{(1)}(x)-\Phi^{(2)} & (x)=t\left(2 F_{1}(x)-F_{0}(x)-F_{2}(x)\right) \\
= & -t \frac{d}{d x}\left(2 U^{\mu_{1}}(x)-U^{\mu_{0}}(x)-U^{\mu_{2}}(x)\right), \quad \text { for } x_{1}<x<x_{2} .
\end{aligned}
$$

We also recall that $2 U^{\mu_{1}}-U^{\mu_{0}}-U^{\mu_{2}}$ vanishes on the support of $\mu_{1}$, and hence in particular at both $x_{1}$ and $x_{2}$. Then the right-hand side is indeed 0 by the fundamental theorem of calculus.

Lemma 5.3 is thus proved, pending the proof of the remarkable identity (5.14). Since the proof of this identity uses new ideas that were not in [11, we decided to give it in a separate lemma.

Lemma 5.4. In the TIS case we have

$$
\int_{y_{2}^{*}}^{y_{3}^{*}}\left(S(x)-\frac{x}{1+x^{2}}\right) d x=\frac{1}{2(r+1)} \int_{x_{1}}^{x_{2}}\left(\Phi^{(1)}(x)-\Phi^{(2)}(x)\right) d x
$$

where $y_{j}^{*}=y_{j}^{\frac{1}{r+1}}$ for $j=2,3$. 
Proof. Consider $\omega=\Phi d z$ as a meromorphic differential on the Riemann surface. Then

$$
\oint_{a} \omega=\int_{x_{1}}^{x_{2}}\left(\Phi^{(1)}(x)-\Phi^{(2)}(x)\right) d x
$$

for the cycle $a$ that goes from $x_{1}$ to $x_{2}$ on the first sheet, and back from $x_{2}$ to $x_{1}$ on the second sheet, cf. Figure 2. The meromorphic differential has simple poles at $-q^{-1}$ on first sheet, at $(-1)^{r} q$ on last sheet, and at $\infty$ with respective residues $1-t, r+t$, and $-1-r$.

Since $z \Phi$ is a degree two meromorphic function we can represent the Riemann surface $\mathcal{R}$ by the equations

$$
\eta^{2}=\prod_{j=0}^{3}\left(\zeta-\zeta_{j}\right), \quad \zeta=z \Phi
$$

with $\zeta_{j}=y_{j} \Phi^{(1)}\left(y_{j}\right)$, for $j=0,1,2,3$, being the four branch points of $\zeta$ with $y_{0}<y_{1}<y_{2}<y_{3}$ by Lemma 4.3 and

$$
0<\zeta_{1}<\zeta_{2}<\zeta_{3}<\zeta_{0}<1
$$

see also Figure 6. In the new coordinates $\mathcal{R}$ is a two sheeted cover of the $\zeta$-plane, with branch cuts $\left[\zeta_{1}, \zeta_{2}\right]$ and $\left[\zeta_{3}, \zeta_{0}\right]$. We label the sheets so that $z=-q^{-1}$ corresponds to $\zeta=\infty$ on the first sheet and $z=(-1)^{r} q$ to $\zeta=\infty$ on the second sheet. Then $\eta$ is positive for real $\zeta>\zeta_{0}$ on the first sheet. The point $z=\infty$ corresponds to the point $\zeta=1$ on the second sheet. The $a$-cycle goes from $\zeta_{2}$ to $\zeta_{3}$ on the first sheet and back from $\zeta_{3}$ to $\zeta_{2}$ on the second sheet.

The meromorphic differential $\omega$ has simple poles at the two points at $\zeta=\infty$ with residues $1-t$ and $r+t$, and at $\zeta=1$ on the second sheet with residue $-1-r$. Then

$$
2 \omega+(r+1) \frac{d \zeta}{\zeta-1}
$$

has residues $\pm(1-2 t-r)$ at the two points at infinity, and residues $\pm(r+1)$ at the two points with $\zeta=1$. Thus (5.17) has an anti-symmetry with respect to the involution $(\zeta, \eta) \mapsto(\zeta,-\eta)$ of $\mathcal{R}$. It follows that

$$
2 \omega+(r+1) \frac{d \zeta}{\zeta-1}=\frac{A \zeta^{2}+B \zeta+C}{\zeta-1} \frac{d \zeta}{\eta}
$$

for certain constants $A, B$, and $C$. From this form we conclude that

$$
\oint_{a}\left(2 \omega+(r+1) \frac{d \zeta}{\zeta-1}\right) d \zeta=2 \int_{\zeta_{2}}^{\zeta_{3}}\left(2 \omega+(r+1) \frac{d \zeta}{\zeta-1}\right) d \zeta
$$


with integration on the first sheet. Clearly $\oint_{a} \frac{d \zeta}{\zeta-1}=0$ and therefore

$$
\begin{aligned}
\oint_{a} \omega & =\int_{\zeta_{2}}^{\zeta_{3}}\left(2 \omega+(r+1) \frac{d \zeta}{\zeta-1}\right) \\
& =2 \int_{y_{2}}^{y_{3}} \Phi^{(1)}(z) d z+(r+1)\left(\log \left(1-\zeta_{3}\right)-\log \left(1-\zeta_{2}\right)\right)
\end{aligned}
$$

since $\left[\zeta_{2}, \zeta_{3}\right]$ on the first sheet corresponds to $\left[y_{2}, y_{3}\right]$ on the first sheet in the original $z$-variable where $\omega=\Phi^{(1)}(z) d z$.

Changing variable $z=x^{r+1}$ and using (2.26) we have

$$
\int_{y_{2}}^{y_{3}} \Phi^{(1)}(z) d z=(r+1) \int_{y_{2}^{*}}^{y_{3}^{*}} S(x) d x
$$

since $y_{j}^{*}=y_{j}^{\frac{1}{r+1}}$. For the last term on the right of 5.18 we recall that $\zeta_{j}=y_{j} \Phi^{(1)}\left(y_{j}\right)$ and $y_{j}$ belongs to $\partial U$. Therefore it satisfies the equation 2.24, that is,

$$
\zeta_{j}=\frac{y_{j}^{\frac{2}{r+1}}}{1+y_{j}^{\frac{2}{r+1}}}=\frac{\left(y_{j}^{*}\right)^{2}}{1+\left(y_{j}^{*}\right)^{2}}, \quad \text { for } j=2,3
$$

which we rewrite as

$$
\log \left(1-\zeta_{j}\right)=-\log \left(1+\left(y_{j}^{*}\right)^{2}\right), \quad \text { for } j=2,3 .
$$

Hence

$$
\begin{aligned}
\log \left(1-\zeta_{2}\right)-\log \left(1-\zeta_{3}\right) & =\log \left(1+\left(y_{3}^{*}\right)^{2}\right)-\log \left(1+\left(y_{2}^{*}\right)^{2}\right) \\
& =2 \int_{y_{2}^{*}}^{y_{3}^{*}} \frac{x}{1+x^{2}} d x
\end{aligned}
$$

Combining (5.15), (5.18), (5.19), (5.21) we obtain the equality of the two integrals in (5.14).

\subsection{Measures $\nu_{t}$ and $\rho_{t}$}

For the proofs of parts (c) and (d), we also need to consider the dynamical picture where we vary $t$, see [11, section 6]. To emphasize the $t$-dependence we attach a subscript $t$ to the notions that vary with $t$. 
We already observed in part (b) that $t \mu_{t}^{*}$ and $t \mu_{\Omega, t}$ increase with $t$. The derivatives

$$
\rho_{t}=\frac{\partial}{\partial t}\left(t \mu_{t}^{*}\right), \quad \nu_{t}=\frac{\partial}{\partial t}\left(t \mu_{\Omega, t}\right)
$$

therefore exist for almost every $t$, as can be proved as in [ $\underline{8}$, Theorem 2], but in our case the derivatives actually exist for every $t \in(0,1)$.

Both $\rho_{t}$ and $\nu_{t}$ are probability measures, with $\operatorname{supp}\left(\rho_{t}\right)=\operatorname{supp}\left(\mu_{t}^{*}\right)$ and $\operatorname{supp}\left(\nu_{t}\right)=\partial \Omega_{t}$, see (2.28). Indeed $\nu_{t}$ measures how the domain $\Omega_{t}$ grows in the spherical metric as $t$ increases.

Applying $\frac{\partial}{\partial t} t$ to the identities (5.3) and (5.4) for the Stieltjes transforms, and using (5.22), we get

$$
\int \frac{d \nu_{t}(s)}{z-s}= \begin{cases}\int \frac{d \rho_{t}(s)}{z-s}, & z \in \mathbb{C} \backslash \Omega_{t}, \\ \frac{z^{r}}{z^{r+1}+q^{-1}}, & z \in \Omega_{t} .\end{cases}
$$

Lemma 5.5. There are constants $C_{1, t}$ and $C_{2, t}$ such that the following hold.

(a) We have

$$
U^{\nu_{t}}(z) \leq U^{\rho_{t}}(z)+C_{2, t}, \quad z \in \mathbb{C},
$$

with equality for $z \in \mathbb{C} \backslash \Omega_{t}$.

(b) We have

$$
U^{\nu_{t}}(z) \leq-\frac{1}{r+1} \log \left|z^{r+1}+q^{-1}\right|+C_{1, t}, \quad z \in \mathbb{C},
$$

with equality for $z \in \Omega_{t}$.

Proof. (a) The first identity in (5.23) implies that $U^{\nu_{t}}-U^{\rho_{t}}$ is constant on each connected component of $\mathbb{C} \backslash \Omega_{t}$. Thus for some constant $C_{2, t}$,

$$
U^{\nu_{t}}(z)=U^{\rho_{t}}(z)+C_{2, t}, \quad z \in \mathbb{C} \backslash \Omega_{t},
$$

since $\mathbb{C} \backslash \Omega_{t}$ is either connected (in BIS and TIS cases), or consists of $r+1$ disjoint components (in FIS case) where due to $r+1$-fold rotational symmetry the constant is the same on each component. If $\Omega_{t}$ is bounded (the BIS case) then $C_{2, t}=0$, since both potentials in $(5.26)$ behave as $-\log |z|+o(1)$ as $z \rightarrow \infty$.

Since $\nu_{t}$ is supported on $\partial \Omega_{t}$, the function $U^{\rho_{t}}-U^{\nu_{t}}$ is superharmonic on the interior of $\Omega_{t}$, including at $\infty$ if $\Omega_{t}$ is unbounded. By the minimum 
principle for superharmonic functions we find the corresponding inequality (5.24) on $\Omega_{t}$, and part (a) follows.

(b) For part (b) we argue similarly, but there is an additional twist when $\Omega_{t}$ is not connected (the TIS case). Using the second identity of (5.23), we apply similar reasoning to $U^{\nu_{t}}$ and $-\frac{1}{r+1} \log \left|z^{r+1}+q^{-1}\right|$, which is the logarithmic potential of the discrete measure with mass $\frac{1}{r+1}$ at each solution of $z^{r+1}+q^{-1}=0$. We find that

$$
U^{\nu t}(z)+\frac{1}{r+1} \log \left|z^{r+1}+q^{-1}\right|
$$

is constant on each connected component of $\Omega_{t}$.

In BIS and FIS cases we have that $\Omega_{t}$ is connected and therefore for some constant $C_{1, t}$,

$$
U^{\nu_{t}}(z)=-\frac{1}{r+1} \log \left|z^{r+1}+q^{-1}\right|+C_{1, t}, \quad z \in \Omega_{t},
$$

in BIS and FIS cases. If $\Omega_{t}$ is unbounded then we let $z \rightarrow \infty$ in (5.27) and we find that $C_{1, t}=0$ in FIS case.

In TIS case we have that $\Omega_{t}$ has two connected components. We find that (5.27) holds with $C_{1, t}=0$ in the unbounded component for the same reason that $C_{1, t}=0$ in FIS case. The bounded component could potentially have a different constant. However we are able to compute $U^{\nu t}(z)$ at $z=0$ because of Lemma 5.3 which says that

$$
t U^{\mu_{\Omega, t}}(0)=-\frac{1-t}{r+1} \log q
$$

in the TIS case. Then taking the $t$-derivative and using the definition 5.22 of $\nu_{t}$, we obtain

$$
U^{\nu_{t}}(0)=\frac{1}{r+1} \log q
$$

which implies that (5.27) with $C_{1, t}=0$ holds for $z=0$ and thus throughout the bounded component as well in the TIS case.

From (5.27) and the fact that $\nu_{t}$ is supported on $\partial \Omega_{t}$, we obtain the inequality (5.25) in all cases (by the minimum principle, as in the proof of part (a)) and part (b) follows.

Remark 5.6. The identity 5.26 and the fact that $\operatorname{supp}\left(\nu_{t}\right)=\partial \Omega_{t}$ show in fact that

$$
\nu_{t}=\operatorname{Bal}\left(\rho_{t}, \partial \Omega_{t}\right) .
$$


Similarly (5.27) gives that

$$
\nu_{t}=\operatorname{Bal}\left(\frac{1}{r+1} \sum_{z^{r+1}=-q^{-1}} \delta_{z}, \partial \Omega_{t}\right) .
$$

Thus $\nu_{t}$ is a balayage measure onto $\partial \Omega_{t}$ from two sides. It is the balayage of $\rho_{t}$ which is supported inside $\Omega_{t}$, and it is also the balayage of a discrete measure supported in the complement on $\Omega_{t}$.

\subsection{Proof of part (c)}

Proof. Integrating the identities $(5.22)$ we obtain the identities

$$
t \mu_{t}^{*}=\int_{0}^{t} \rho_{s} d s, \text { and } t \mu_{\Omega, t}=\int_{0}^{t} \nu_{s} d s
$$

which are analogous to the formulas of Buyarov and Rakhmanov [8] for varying families of measures on the real line. We also have

$$
\begin{aligned}
t \mu_{\Omega, t} & =\lim _{\tau \rightarrow 1-} \tau \mu_{\Omega, \tau}-\int_{t}^{1} \nu_{s} d s \\
& =\frac{d A(z)}{\pi\left(1+|z|^{2}\right)^{2}}-\int_{t}^{1} \nu_{s} d s .
\end{aligned}
$$

We can calculate the logarithmic potential

$$
-\int_{\mathbb{C}} \log |z-s| \frac{d A(s)}{\pi\left(1+|s|^{2}\right)}=-\frac{1}{2} \log \left(1+|z|^{2}\right), \quad z \in \mathbb{C} .
$$

Therefore by (5.29) and (5.25), we have for every $z \in \mathbb{C}$,

$$
\begin{aligned}
t U^{\mu_{\Omega, t}}(z) & =-\frac{1}{2} \log \left(1+|z|^{2}\right)-\int_{t}^{1} U^{\nu_{s}}(z) d s \\
& \geq-\frac{1}{2} \log \left(1+|z|^{2}\right)+\int_{t}^{1}\left(\frac{1}{r+1} \log \left|z^{r+1}+q^{-1}\right|-C_{1, s}\right) d s \\
& =-\frac{1}{2} \log \left(1+|z|^{2}\right)+\frac{1-t}{r+1} \log \left|z^{r+1}+q^{-1}\right|+t c_{1, t}
\end{aligned}
$$

with $c_{1, t}=-\frac{1}{t} \int_{t}^{1} C_{1, s} d s$.

Equality holds in 5.25 for $z \in \Omega_{t}$ which implies that equality holds in (5.30) for

$$
z \in \bigcap_{t \leq s<1} \Omega_{s}=\Omega_{t}
$$

since $\Omega_{t} \subset \Omega_{s}$ whenever $t<s$. The proof of part (c) is complete. 


\subsection{Proof of part (d)}

Proof. We obtain for every $z \in \mathbb{C}$, using (5.28) and (5.24),

$$
\begin{aligned}
t U^{\mu_{\Omega, t}}(z) & =\int_{0}^{t} U^{\nu_{s}}(z) d s \\
& \leq \int_{0}^{t}\left(U^{\rho_{s}}(z)+C_{2, s}\right) d s \\
& =t U^{\mu_{t}^{*}}(z)+t c_{2, t} \quad \text { with } c_{2, t}=\frac{1}{t} \int_{0}^{t} C_{2, s} d s .
\end{aligned}
$$

Equality holds, by Lemma 5.5 (a), for

$$
z \in \bigcap_{0<s \leq t}\left(\mathbb{C} \backslash \Omega_{s}\right)=\mathbb{C} \backslash \Omega_{t}
$$

since $\Omega_{s} \subset \Omega_{t}$ whenever $s<t$.

\section{References}

[1] D. Aharonov and H.S. Shapiro, Domains on which analytic functions satisfy quadrature identities, J. Anal. Math. 30 (1976), 39-73.

[2] A.I. Aptekarev and A.B.J. Kuijlaars, HermitePadé approximations and multiple orthogonal polynomial ensembles, Uspekhi Mat. Nauk. 66 (6) (2011) 123-190; English transl. in Russian Math. Surveys 66 (2011) $1133-1199$.

[3] F. Balogh, M. Bertola, S.Y. Lee and K.D.T-R. McLaughlin, Strong asymptotics of the orthogonal polynomials with respect to a measure supported on the plane. (English summary) Comm. Pure Appl. Math. 68 (2015), no. 1, 112172.

[4] P.M. Bleher and A.B.J. Kuijlaars, Orthogonal polynomials in the normal matrix model with a cubic potential, Adv. Math. 230 (2012), 12721321.

[5] P.M. Bleher and G.L.F. Silva, The mother body phase transition in the normal matrix model, Mem. Amer. Math. Soc. 265 (2020), no. 1289.

[6] A. Borodin, Biorthogonal ensembles, Nucl. Phys. B 536 (1999), 704732 . 
[7] J. Brauchart, P. Dragnev, E.B. Saff, and R. Womersley, Logarithmic and Riesz equilibrium for multiple sources on the sphere: the exceptional case, in: Contemporary Computational Mathematics (J. Dick, F.Y. Kuo, and H. Wozniakowski, eds.), Springer, Cham, 2018, pp. 179 203.

[8] V.S. Buyarov and E.A. Rakhmanov, Families of equilibrium measures in an external field on the real axis, Sb. Math. 190 (1999), 791-802.

[9] T. Claeys, M. Girotti, and D. Stivigny, Large gap asymptotics at the hard edge for product random matrices and Muttalib-Borodin ensembles, Int. Math. Res. Not. IMRN 2019, no. 9, 2800-2847.

[10] T. Claeys and S. Romano, Biorthogonal ensembles with two-point interactions, Nonlinearity 27 (2014), 2419-2444.

[11] J.G. Criado del Rey and A.B.J. Kuijlaars, An equilibrium problem on the sphere with two equal charges, preprint arXiv:1907.04801.

[12] D. Crowdy, Quadrature domains and fluid dynamics, in: Quadrature domains and their applications, Oper. Theory Adv. Appl., vol. 156, Birkhäuser, Basel, 2005, 113-129.

[13] D. Crowdy and M. Cloke, Analytical solutions for distributed multipolar vortex equilibria on a sphere, Phys. Fluids 15 (2003), 22-34.

[14] P. Deift, T. Kriecherbauer, and K.T-R. McLaughlin, New results on the equilibrium measure for logarithmic potentials in the presence of an external field, J. Approx. Theory 95 (1998), 388-475.

[15] P.D. Dragnev, Constrained energy problems for logarithmic potentials, Ph.D. Thesis, University of South Florida, Tampa, FL, 1997.

[16] P.D. Dragnev, On the separation of logarithmic points on the sphere, in: Approximation Theory X (L.L. Schumaker, C.K. Chui, and J. Stöckler, eds.) Vanderbilt Univ. Press, Nashville, TN, 2002, pp. 137-144.

[17] M. Duits and A.B.J. Kuijlaars, An equilibrium problem for the limiting eigenvalue distribution of banded Toeplitz matrices, SIAM J. Matrix Anal. Appl. 30 (2008) 173-196.

[18] P. Elbau and G. Felder, Density of eigenvalues of random normal matrices, Comm. Math. Phys. 259 (2005), 433-450. 
[19] P.J. Forrester, D-Z. Liu, and P. Zinn-Justin, Equilibrium problems for Raney densities, Nonlinearity 28 (2015), 2265-2277.

[20] P.J. Forrester and D. Wang, Muttalib-Borodin ensembles in random matrix theory-realisations and correlation functions. Electron. J. Probab. 22 (2017), Paper No. 54, 43 pp.

[21] A.A. Gonchar and E.A. Rakhmanov, On convergence of simultaneous Padé approximants for systems of functions of Markov type, Trudy Mat. Inst. Steklov 157 (1981) 31-48; English transl. in Proc. Steklov Inst. Math. 157 (1983) 31-50.

[22] A.A. Gonchar and E.A. Rakhmanov, On the equilibrium problem for vector potentials, Uspekhi Mat. Nauk 40 (4) (1985) 155-156; English transl. Math. Surveys 40 (4) (1985) 183-184.

[23] B. Gustafsson, Lectures on Balayage, Univ. Joensuu Dept. Math. Rep. Ser. 7, Univ. Joensuu, Joensuu, 2004, pp. 17-63.

[24] B. Gustafsson and H.S. Shapiro, What is a quadrature domain?, in Quadrature Domains and Their Applications, Oper. Theory Adv. Appl., vol. 156, Birkhäuser, Basel, 2005, pp. 1-25,

[25] B. Gustafsson, R. Teoderscu, and A. Vasil'ev, Classical and stochastic Laplacian growth, Birkhäuser Verlag, Basel, 2014.

[26] B. Gustafsson and V.G. Tkachev, On the exponential transform of lemniscates, Comp. Methods Function Theory 11 (2011), 591-615.

[27] A. Hardy and A.B.J. Kuijlaars, Weakly admissible vector equilibrium problems, J. Approx. Theory 164 (2012), 854-868.

[28] A.B.J. Kuijlaars, Multiple orthogonal polynomials in random matrix theory, in: Proceedings of the International Congress of Mathematicians, Volume III (R. Bhatia, ed.) Hyderabad, India, 2010, pp. 14171432 .

[29] A.B.J. Kuijlaars, A vector equilibrium problem for Muttalib-Borodin biorthogonal ensembles, SIGMA Symmetry Integrability Geom. Methods Appl. 12 (2016), Paper No. 065, 15 pp.

[30] A.B.J. Kuijlaars and P.D. Dragnev, Equilibrium problems associated with fast decreasing polynomials, Proc. Amer. Math. Soc. 127 (1999), 1065-1074. 
[31] A.B.J. Kuijlaars and A. López García, The normal matrix model with a monomial potential, a vector equilibrium problem, and multiple orthogonal polynomials on a star, Nonlinearity 28 (2015), 347-406.

[32] A.B.J. Kuijlaars and L.D. Molag, The local universality of MuttalibBorodin biorthogonal ensembles with parameter $\theta=\frac{1}{2}$, Nonlinearity 32 (2019), 3023-3081.

[33] A.B.J. Kuijlaars and A. Tovbis, The supercritical regime in the normal matrix model with cubic potential, Adv. Math. 283 (2015), 530-587.

[34] S.Y. Lee and N. Makarov, Topology of quadrature domains, J. Amer. Math. Soc. 29 (2016), 333-369.

[35] S.Y. Lee, R. Teodorescu, and P. Wiegmann, Shocks and finite-time singularities in Hele-Shaw flow, Phys. D 238 (2009), 1113-1128.

[36] S.Y. Lee, R. Teodorescu, and P. Wiegmann, Weak solution of the HeleShaw problem: shocks and viscous fingering, JETP Lett. 92 (2010), 91-96.

[37] S.Y. Lee, R. Teodorescu, and P. Wiegmann, Viscous shocks in HeleShaw flow and Stokes phenomena of the Painlevé I transcendent, Phys. D 240 (2011), 1080-1091.

[38] S.Y. Lee and M. Yang, Discontinuity in the asymptotic behavior of planar orthogonal polynomials under a perturbation of the Gaussian weight, Comm. Math. Phys. 355 (2017), 303-338.

[39] A.R. Legg and P.D. Dragnev, Logarithmic equilibrium on the sphere in the presence of multiple point charges, preprint arXiv:1912.11013.

[40] L.D Molag, The local universality of Muttalib-Borodin ensembles when the parameter $\theta$ is the reciprocal of an integer, preprint arXiv:2003.11299.

[41] K.A. Muttalib, Random matrix models with additional interactions, J. Phys. A: Math. Gen. 28 (1995), L159-64.

[42] E.M. Nikishin and V.N. Sorokin, Rational Approximations and Orthogonality, Amer. Math. Soc., Providence, RI, 1991.

[43] R. Orive, J.F. Sánchez Lara, and F. Wielonsky, Equilibrium problems in weakly admissible external fields created by point charges, J. Approx. Theory 244 (2019), 71-100. 
[44] T. Ransford, Potential Theory in the Complex Plane, Cambridge Univ. Press, Cambridge, 1995.

[45] E.B. Saff and V. Totik, Logarithmic Potentials with External Fields, Springer-Verlag, Berlin, 1997.

[46] W. Schlag, A Course in Complex Analysis and Riemann Surfaces, Amer. Math. Society, Providence, RI, 2014.

[47] P. Simeonov, A weighted energy problem for a class of admissible weights, Houston J. Math. 31 (2005), 1245-1260.

[48] R. Teodorescu, E. Bettelheim, O. Agam, A. Zabrodin, and P. Wiegmann, Normal random matrix ensemble as a growth problem. Nuclear Phys. B 704 (2005), 407-444. 